The University of Maine

\title{
DigitalCommons@UMaine
}

Marine Sciences Faculty Scholarship

School of Marine Sciences

$12-1-2002$

\section{Kelp Forest Ecosystems: Biodiversity, Stability, Resilience and Future}

Robert Steneck

University of Maine - Main, steneck@maine.edu

M. H. Graham

B. J. Bourque

D. Corbett

J.M. Erlandson

See next page for additional authors

Follow this and additional works at: https://digitalcommons.library.umaine.edu/sms_facpub

\section{Repository Citation}

Steneck, Robert; Graham, M. H.; Bourque, B. J.; Corbett, D.; Erlandson, J. M.; Estes, J. A.; and Tegner, M. J., "Kelp Forest Ecosystems: Biodiversity, Stability, Resilience and Future" (2002). Marine Sciences Faculty Scholarship. 65.

https://digitalcommons.library.umaine.edu/sms_facpub/65

This Review is brought to you for free and open access by DigitalCommons@UMaine. It has been accepted for inclusion in Marine Sciences Faculty Scholarship by an authorized administrator of DigitalCommons@UMaine. For more information, please contact um.library.technical.services@maine.edu. 
Authors

Robert Steneck, M. H. Graham, B. J. Bourque, D. Corbett, J. M. Erlandson, J. A. Estes, and M. J. Tegner 


\title{
Kelp forest ecosystems: biodiversity, stability, resilience and future
}

\author{
ROBERT S. STENECK ${ }^{1 *}$, MICHAEL H. GRAHAM ${ }^{2}$, BRUCE J. BOURQUE ${ }^{3}$, DEBBIE \\ CORBETT $^{4}, \mathrm{JON}_{\text {M. ERLANDSON }}^{5}$, JAMES A. ESTES ${ }^{6}$ AND MIA J. TEGNER TA $^{7}$ \\ ${ }^{1}$ School of Marine Sciences, University of Maine, Darling Marine Center, Walpole, ME 04573, USA, ${ }^{2}$ Center for Population Biology, University \\ of California, Davis, One Shields Avenue, Davis, CA 95616, USA, ${ }^{3}$ Department of Anthropology, Bates College, Lemiston, ME 04240, USA, \\ ${ }^{4}$ US Fish and Wildlife Service, 1011 East Tudor Road, Anchorage, AK 99503-6119, USA, ${ }^{5}$ Department of Anthropology, University of Oregon, \\ 308 Condon Hall, Eugene, OR 97403-1218, USA, ${ }^{6}$ US Geological Survey, Long Marine Laboratory, 100 Shaffer Road, University of \\ California, Santa Cruz, CA 95060, USA and ${ }^{7}$ Scripps Institution of Oceanography, University of California, San Diego, La Folla, CA \\ 92093-0201, USA \\ $\uparrow$ Deceased \\ Date submitted: 7 November 2001 Date accepted: 25 September 2002
}

\section{SUMMARY}

Kelp forests are phyletically diverse, structurally complex and highly productive components of coldwater rocky marine coastlines. This paper reviews the conditions in which kelp forests develop globally and where, why and at what rate they become deforested. The ecology and long archaeological history of kelp forests are examined through case studies from southern California, the Aleutian Islands and the western North Atlantic, well-studied locations that represent the widest possible range in kelp forest biodiversity. Global distribution of kelp forests is physiologically constrained by light at high latitudes and by nutrients, warm temperatures and other macrophytes at low latitudes. Within mid-latitude belts (roughly $40-60^{\circ}$ latitude in both hemispheres) well-developed kelp forests are most threatened by herbivory, usually from sea urchins. Overfishing and extirpation of highly valued vertebrate apex predators often triggered herbivore population increases, leading to widespread kelp deforestation. Such deforestations have the most profound and lasting impacts on species-depauperate systems, such as those in Alaska and the western North Atlantic. Globally urchininduced deforestation has been increasing over the past 2-3 decades. Continued fishing down of coastal food webs has resulted in shifting harvesting targets from apex predators to their invertebrate prey, including kelp-grazing herbivores. The recent global expansion of sea urchin harvesting has led to the widespread extirpation of this herbivore, and kelp forests have returned in some locations but, for the first time, these forests are devoid of vertebrate apex predators. In the western North Atlantic, large predatory crabs have recently filled this void and they have become the new apex predator in this system. Similar shifts from fish- to crab-dominance may have occurred in coastal zones of the United Kingdom and Japan, where large predatory finfish were extirpated long ago. Three
North American case studies of kelp forests were examined to determine their long history with humans and project the status of future kelp forests to the year 2025. Fishing impacts on kelp forest systems have been both profound and much longer in duration than previously thought. Archaeological data suggest that coastal peoples exploited kelp forest organisms for thousands of years, occasionally resulting in localized losses of apex predators, outbreaks of sea urchin populations and probably small-scale deforestation. Over the past two centuries, commercial exploitation for export led to the extirpation of sea urchin predators, such as the sea otter in the North Pacific and predatory fishes like the cod in the North Atlantic. The largescale removal of predators for export markets increased sea urchin abundances and promoted the decline of kelp forests over vast areas. Despite southern California having one of the longest known associations with coastal kelp forests, widespread deforestation is rare. It is possible that functional redundancies among predators and herbivores make this most diverse system most stable. Such biodiverse kelp forests may also resist invasion from non-native species. In the species-depauperate western North Atlantic, introduced algal competitors carpet the benthos and threaten future kelp dominance. There, other non-native herbivores and predators have become established and dominant components of this system. Climate changes have had measurable impacts on kelp forest ecosystems and efforts to control the emission of greenhouse gasses should be a global priority. However, overfishing appears to be the greatest manageable threat to kelp forest ecosystems over the 2025 time horizon. Management should focus on minimizing fishing impacts and restoring populations of functionally important species in these systems.

Keymords: apex predators, biodiversity, herbivory, human interactions, kelp forests, trophic cascades

* Correspondence: Professor Robert Steneck Tel: + 12075633146 ext. 233 Fax: +1 2075633119 e-mail: steneck@maine.edu 


\section{INTRODUCTION}

'I can only compare these great aquatic forests ... with the terrestrial ones in the intertropical regions. Yet if in any country a forest was destroyed, I do not believe nearly so many species of animals would perish as would here, from the destruction of the kelp. Amidst the leaves of this plant numerous species of fish live, which nowhere else could find food or shelter; with their destruction the many cormorants and other fishing birds, the otters, seals, and porpoise, would soon perish also; and lastly, the Fuegian[s] ... would ... decrease in numbers and perhaps cease to exist.'

Charles Darwin, 1 June 1834, Tierra del Fuego, Chile (Darwin 1909, pp. 256-257).

Kelp forests dominate shallow rocky coasts of the world's cold-water marine habitats. They comprise primarily brown algae in the order Laminariales and produce the largest biogenic structures found in benthic marine systems (Dayton 1985a). Kelp forest ecosystems include structure-producing kelps and their myriad associated biota such as marine mammals, fishes, crabs, sea urchins, molluscs, other algae and epibiota that collectively make this one of the most diverse and productive ecosystems of the world (Mann 1973). Economically, kelp forest ecosystems have been significant to maritime peoples for thousands of years (Simenstad et al. 1978; Erlandson 2001).

Past reviews provided contemporary overviews of kelp forests (Kain 1979; Dayton 1985a; Schiel \& Foster 1986; Witman \& Dayton 2001). These rarely considered how kelp forest ecosystems have changed at very large spatial scales over very long periods of time spanning decades to millennia (Jackson et al. 2001). A longer historical perspective allows us to see how kelp forest ecosystems have changed and possibly where they are headed in the future. Thus, it is with larger spatial and temporal perspective that we embarked on this review.

\section{The nature of kelp}

Three morphological groups or 'guilds' of kelp are defined by the canopy height of their fronds (Dayton 1985a). 'Canopy' kelps are largest and produce floating canopies. Chief among these is the giant kelp, Macrocystis spp. which grows to $45 \mathrm{~m}$ long (Abbott \& Hollenberg 1976) and dominates kelp forests along the west coasts of North and South America and at scattered locations in the South Pacific Ocean including South Africa, southern Australia, New Zealand and several subantarctic islands (Fig. 1). Smaller canopy kelps include Nereocystis leutkeana, which ranges from Central California to Alaska, and its Southern Hemisphere counterpart, Ecklonia maxima in South Africa, and Alaria fistulosa in Alaska and the Pacific coast of Asia. These kelps reach about $10 \mathrm{~m}$ in length. 'Stipitate' kelp fronds are held above the benthos with rigid stipes (Dayton 1985a). They include some species of Laminaria sp. in Europe and the Pacific Northwest, Ecklonia in southern Australia and New Zealand, and Lessonia in Chile. Stipitate Laminaria dominates kelp forests of the North Pacific from Japan, north-east across coastal Alaska to northern California. Most species of Laminaria are less than $5 \mathrm{~m}$ long, but a few grow to $10 \mathrm{~m}$ in length. Other stipitate genera found along the Pacific coast of North America include Pterygophora, Eisenia, Pleurophycus and
Figure 1 Kelp forest distributions of the world and their dominant genera (from Raffaelli \& Hawkins 1996).

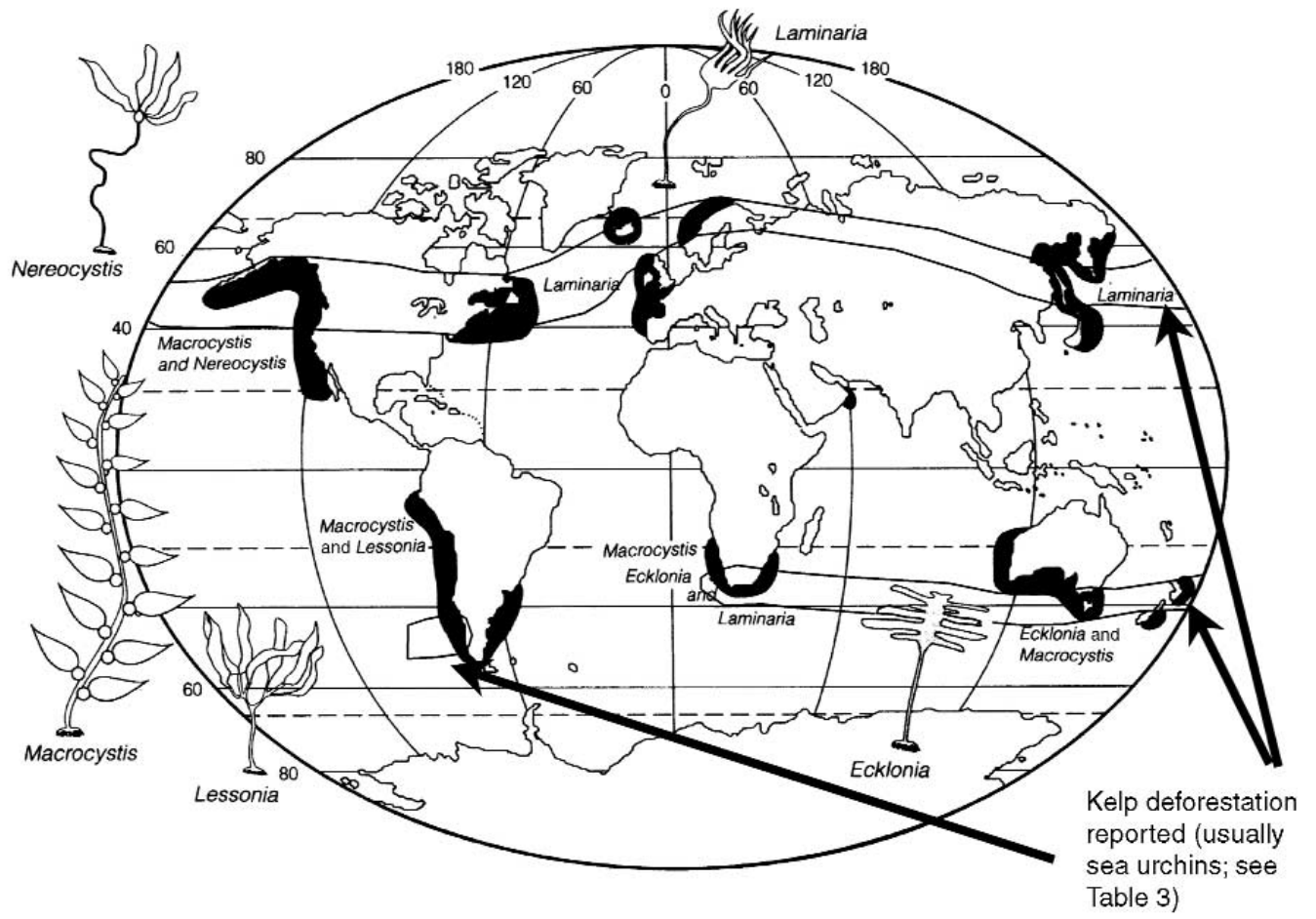


Thalassiophyllum. 'Prostrate' kelps are most diminutive and cover the benthos with their fronds. This guild includes several species of Laminaria, which dominate most of the Northern Hemisphere except for parts of the eastern North Pacific (Fig. 1). Prostrate Laminaria forests range from the Gulf of Maine to Greenland in the western North Atlantic and from Iceland to the high Arctic of Norway and south to the north-westernmost corner of Africa, in the eastern North Atlantic. Taxonomically, the kelps themselves are not diverse. The most diverse kelp flora occurs along the California coast of North America. However, it only has 20 species of kelp distributed among 16 genera because most genera are monotypic (Abbott \& Hollenberg 1976).

Despite their low taxonomic diversity, kelps are highly diverse structurally and functionally. They possess specialized cells for translocation (for example, 'trumpet hyphae' and sieve elements) that allow them to attain their great size and diverse morphology. Even within species, developmental stages and ploidy-phases of kelp span an unparalleled range of sizes and shapes. Their gametophyte stage is an inconspicuous, microscopic filament living within the benthic boundary layer (Neushul 1972). In this microhabitat fertilization occurs and developing sporophytes grow. Depending on the adult morphology of the species, the kelp fronds may remain near the benthos (prostrate forms), occupy intermediate depths (stipitate forms), or grow to and float on the surface (canopy forms) (Neushul 1972). Morphology varies widely among and within genera. The tiering of kelp morphologies along with other associated taxa contributes to the structural diversity of this system. All three kelp forms can coexist (Dayton 1985a) together with a patchy carpet of corticated macrophyte turf (Dayton 1975) and a pavement of encrusting coralline algae. Each of these structural components is habitat and food for associated organisms. Thus the unique anatomy of kelp allows communities to attain the stature of forests with wide-ranging implications for associated organisms and coastal communities.

The structure and function of kelp forests differ significantly from their terrestrial counterparts dominated by angiosperm and gymnosperm trees. Compared to terrestrial forests, kelp forests are more productive and diverse (at the phyla level), but the average lifespan and structural height is less. For example, many mature terrestrial forests attain canopy heights of $10-30 \mathrm{~m}$ within 20 to 30 years. Individual trees can have longevities of centuries to millennia. Associated with terrestrial forests are animals primarily from the three phyla Chordata, Arthropoda and Annelida. In contrast, kelp forests reach canopy heights of $1-15 \mathrm{~m}$ (although Macrocystis is larger than this) within 1-3 years. Individual kelps have maximum longevities of about 25 years (Steneck \& Dethier 1994). Associated with kelp forests are animals from more than 10 phyla, namely Chordata, Arthropoda, Annelida, Echinodermata, Bryozoa, Cnidaria, Mollusca, Platyhelminthes, Brachiopoda and Porifera.

The physical structure, algal biomass and organisms associated with kelp forests profoundly alter local environ- ments and ecologies. Kelp canopies dampen waves, which influence water flow and associated processes of coastal erosion, sedimentation, benthic productivity (primary and secondary) and recruitment (Duggins et al. 1990). The canopies also reduce light, creating understorey conditions favourable for a suite of species adapted to low light intensity (Santelices \& Ojeda 1984a); as a result, they can influence interspecific competition among algae (Dayton 1985a). Kelps are substratum for numerous sessile animals and algae (Duggins 1980; Reed \& Foster 1984; Dunton \& Shell 1987) and habitat for mobile organisms specialized to live and feed directly on the kelp or its associated assemblages. For example, trophically specialized limpets depend upon kelp for their existence (Steneck \& Watling 1982; Estes \& Steinberg 1988; Bustamante et al. 1995). Kelp forest architecture provides habitat, nursery ground and food for myriad mobile pelagic and benthic organisms (Bernstein \& Jung 1980; Bologna \& Steneck 1993; Levin 1994; Anderson et al. 1997). Since predatory fishes use canopies as habitat, canopy loss can translate to increased survivorship of resident prey organisms and their larvae (Gaines \& Roughgarden 1987). Thus, as kelp canopies vary, the ecological and oceanographic processes associated with them will be altered.

Kelps concentrate biomass and are a significant source of nutrition for coastal marine ecosystems via food webs based on macroalgal detritus (Duggins et al. 1989) because herbivores rarely consume more than $10 \%$ of the living biomass (Mann 2000). Large pieces of kelp commonly litter the benthos in coastal zones and become food for detritivores and microbes (Linley et al. 1981), thus making their carbon available to the coastal community of suspension feeders as well as the herbivores and detritivores feeding directly on kelps (Dunton \& Schell 1987; Duggins et al. 1989). In effect, kelp forests concentrate and magnify secondary production, thereby supporting complex food webs in coastal zones (Duggins et al. 1989; Mann 2000).

The combination of high kelp productivity in environments with the potential for high rates of disturbance can make these forests surprisingly ephemeral. Entire kelp beds can be eliminated by thermal events, storms or outbreaks of herbivores and disappear without a trace within a year, but significantly, the entire community can return nearly as quickly (Scheibling 1984; Harrold \& Reed 1985; Hart \& Scheibling 1988; Witman 1988; Tegner et al. 1997).

As with most ecosystems, kelp forests are strongly influenced by both their physical and biological environment. Fortunately, we know much more about how environmental forcing functions operate in kelp forests than we do for most other ecosystems. Kelp forests are ideally located and scaled for human study; most components of the system are readily observable, kelp forest phase-changes are rapid (i.e. occurring within a few years) and manipulative experiments of canopy removal or transplantation are easily accomplished. Thus, hypotheses can be advanced, tested, reported and challenged within a decade. 


\section{LARGE SCALE PATTERNS: GLOBAL INTERPLAY OF DIVERSITY, PRODUCTIVITY AND DISTURBANCE}

\section{The ecology of kelp forests}

Kelp forests persist in a balance between ecological processes driving their development and their deforestation. They wax as a result of recruitment and net productivity and wane as a result of biomass lost from disturbances both physical and biological and competition. These factors are influenced by properties intrinsic to, and extrinsic of, the kelp (Steneck \& Dethier 1994). Complex, multifactorial interactions make long-term changes in these systems difficult to demonstrate and predict (Tegner et al. 1996a). Further, the population density and community development in structurally diverse (high canopy) kelp forests may decouple changes in the canopy cover from changes in population densities, thus obscuring linkages between patterns and processes (Schiel \& Foster 1986; Dayton et al. 1998). Nevertheless, several strong ecological interactions control forest development and deforestation. These operate at varying spatial and temporal scales and they will be the focus of this section. For this, we will consider where kelps can live, where they develop forests, and under what conditions kelp deforestation takes place.

\section{Kelp forest development}

Globally, kelp forests develop on shallow rocky shores in a mid-latitude band where light and oceanographic conditions allow the development and persistence of this growth form (Fig. 1). While kelps can grow in Arctic and sub-Antarctic regions (see Dunton \& Dayton 1995), their abundance and diversity are low (probably due to light limitations; Dunton 1990; Henley \& Dunton 1997) and thus they rarely develop forests above about $60^{\circ}$ latitude. Similarly, warm temperatures and low nutrient concentrations generally prevent kelp forests from developing in subtropical or tropical regions (Bolton \& Anderson 1987; Gerard 1997). The lowest latitude kelp beds (see Hatcher et al. 1987) usually correspond with ocean current driven anomalies in latitudinal gradients of warm temperatures and/or low nutrient conditions. For example, kelp forests are found within the tropics of Cancer and Capricorn only along the western coasts of southern California to Mexico, northern Chile to Peru, western South Africa and western Australia (Fig. 1). In these cases, cool ocean currents flowing toward the equator, or upwelling, advect cool, nutrient-rich water to the kelp forests.

Low latitude kelp (usually less than $40^{\circ}$ latitude) are often diminutive and share or lose community dominance to fucoids such as Sargassum and other large brown algae that become more diverse and abundant toward the tropics. In North America, southern California kelps share space with the fucoids Cystoseira osmundacea $\left(40-30^{\circ} \mathrm{N}\right.$ latitude) and Sargassum spp. (35-25N N latitude) (Foster \& Schiel 1985). At Japan's southern island, Honshu, scores of Sargassum species become increasingly important and eventually dominate macroalgal communities at latitudes less than about $40^{\circ} \mathrm{N}$ (Fujita 1998). One of the northernmost kelp of the Southern Hemisphere is in Western Australia at $28-29^{\circ} \mathrm{S}$ latitude (Hatcher et al. 1987), where diminutive Ecklonia share space with brown algal species of Lobophora and Sargassum. Elsewhere in South Australia (Shepherd \& Womersley 1970) and New Zealand (Choat \& Schiel 1982), large subtidal fucoids cohabit with and often dominate the kelp. In New Zealand, fucoid densities were twice that of kelp densities at low latitude $\left(<40^{\circ} \mathrm{S}\right)$ sites, but reversed to kelp dominance at the higher latitude site $\left(>40^{\circ} \mathrm{S}\right)$, where kelp densities were twice that of the fucoids (Choat \& Schiel 1982). New Zealand fucoids frequently dominate shallow zones (upper $5 \mathrm{~m}$ ), displacing kelp dominance to below $10 \mathrm{~m}$ (Choat \& Schiel 1982). Only the relatively low diversity kelp forests of South Africa $\left(22-35^{\circ} \mathrm{S}\right.$ latitude) and northern Chile (40-20 ${ }^{\circ} \mathrm{S}$ latitude) are without fucoids (Bolton 1996). However, in Chile the large kelp-like Durvillaea sp. (closely related to the fucoids) commonly dominates shallow zones (Santelices 1990) and may outcompete Macrocystis there (Dayton 1985b).

In general, the three interacting processes that control the development of kelp forests are recruitment, growth and competition. Locally, kelp forests are established and maintained by successful settlement of zygotes, which grow and are thinned by mortality from intraspecific competition during their benthic life (Reed \& Foster 1984; Chapman 1986). Recruitment is often seasonal and influenced by environmental conditions at the time of settlement. In complex kelp forests tiered with multiple levels (for example, canopy, stipitate and prostrate forms; Table 1) such as the California kelp forest, kelp recruitment and growth is regulated by light available through breaks in the kelp canopy (Reed \& Foster 1984; Santelices \& Ojeda 1984a; Graham et al. 1997) as well as by available nutrients (Dayton et al. 1999). Following intense storms that deforest or thin kelp canopies, recruitment is usually strong, but the kelp species that grow to dominance will depend upon nutrient conditions at the time (Tegner et al. 1997).

Kelp growth depends on interactions among nutrient availability, temperature and light. Kelps dominate cold-water coastal zones (Fig. 1) but can become physiologically stressed at high sea temperatures, particularly when nutrient availability is low (Tegner et al. 1996a; Gerard 1997). In some regions without upwelling, periods of low nutrient concentrations correspond with warm summer temperatures when the water is stratified. The combined effects of low nutrients and high rates of respiration result in kelp plants that erode more rapidly than they grow (Gagne et al. 1982; R.S. Steneck, unpublished data 2002). In kelp forests driven by the upwelling of new nitrogen, such as those of southern California, warm surface water temperature is a surrogate for low nutrient availability (Tegner et al. 1996a). In this system when El Niño events disrupt coastal upwelling, kelp becomes nutrientstarved and dies back (Tegner \& Dayton 1991). As a result, the distribution, abundance and size of kelp plants decline as sea surface temperatures increase (Dayton et al. 1999). 
Table 1 Dominant organisms, functional groups and diversity that define the structure and function of subtidal kelp forest ecosystems of North America. *Steller's sea cow (now extinct).

\begin{tabular}{|c|c|c|c|}
\hline & Western North Atlantic & Aleutians, Alaska & Southern California \\
\hline Dominant kelp genera & Laminaria (1 sp.), Agarum (1 sp.) & $\begin{array}{l}\text { Alaria (1 sp.), Laminaria (3 spp.), } \\
\text { Thalassiophyllum (1 sp.), } \\
\text { Agarum (1 sp.) }\end{array}$ & $\begin{array}{l}\text { Macrocystis (1 sp.), Pterygophora } \\
\text { (1 sp), Laminaria (1 sp.), Eisenia } \\
\text { (1 sp.), Pelagophycus (1 sp.), } \\
\text { Egregia (1 sp.), Agarum (1 sp.) }\end{array}$ \\
\hline $\begin{array}{l}\text { Structural tiering } \\
\quad \text { (kelp guilds) }\end{array}$ & Prostrate & Canopy, stipitate, prostrate & Canopy, stipitate, prostrate \\
\hline \multicolumn{4}{|l|}{ Dominant herbivores } \\
\hline Sea urchins & Strongylocentrotus droebachiensis & Strongylocentrotus polycanthus & $\begin{array}{l}\text { Lytechinus anamesus, } \\
\text { Strongylocentrotus purpuratus, } \\
\text { S. franciscanus }\end{array}$ \\
\hline Molluscs & & & Haliotis (3 spp.), Tegula (3 spp.) \\
\hline Fishes & & & $\begin{array}{l}\text { Medialuna californiesis, Girella } \\
\text { nigricanus }\end{array}$ \\
\hline Marine mammals & & Hydrodamalis gigas* & Hydrodamalis gigas* \\
\hline Dominant carnivores & Atlantic cod (Gadhus morhua) & $\begin{array}{l}\text { Sea otter (Enhydra lutris), killer } \\
\quad \text { whales (Orcinus orca) }\end{array}$ & $\begin{array}{l}\text { Sea otter, spiny lobster (Panulirus } \\
\text { interuptus), sheephead fish } \\
\text { (Semicossyphus pulcher) }\end{array}$ \\
\hline
\end{tabular}

As a group, kelps have a relatively low photosynthetic to biomass ratio. This constrains them to relatively shallow, well-illuminated zones compared to other functional groups of algae (reviewed in Vadas \& Steneck 1988). In environments free of herbivores or other agents of disturbance, kelp frond size and density decline rapidly with depth making kelps the shallowest of the major growth forms of marine algae (Steneck \& Dethier 1994). Extended periods of darkness in the Arctic limit the northern distribution (Fig. 1), diversity and maximum depth of kelps (Henley \& Dunton 1997).

The limited development of kelps at high latitudes results from interactions among light, temperature, ice scour, nutrients and evolutionary biogeography. As a result, true kelps (order Laminariales) grow much further north in the Arctic (to about $70^{\circ}$ North latitude) than they do south in the Antarctic (to $55^{\circ}$ South latitude) (Dunton \& Dayton 1995). Kelp growth in near-freezing seawater temperatures requires dissolved inorganic nitrogen to physiologically accommodate photosynthesis (Korb \& Gerard 2000). The endemic Arctic kelp Laminaria solidungula has a remarkable capacity to store nitrogen that is only available in the winter when it has no light (Henley \& Dunton 1997). In contrast, in the Antarctic where nitrogen is available all year round, true kelps are displaced by morphologically similar brown algae, Himantothallus sp. of the order Desmarestiales (Moe \& Silva 1981; Dunton \& Dayton 1995). This kelp-like alga has no inherent capacity to store nitrogen, nor does it need to in the nitrogen-replete waters of the Antarctic. Under experimental nitrogen-starved conditions this alga quickly succumbs (Korb \& Gerard 2000). Thus Arctic and Antarctic distributions of kelp may hinge on physiological adaptations to light and nutrient limitations in those areas. Arctic kelp illustrates the remarkable range of physiological tolerance that can evolve in the group. The effective replacement of
Laminariales in the high Antarctic by large brown algae in the order Desmarestiales may be an evolutionary priority effect of an older order in the geologically older Southern Hemisphere ocean system. Nevertheless, polar populations of kelps or kelp growth forms may grow to their physiological limits at high latitudes but they do not attain forest there. Seasonal change in oceanography and physical disturbances from ice scour can limit or eliminate subarctic populations of kelps (Himmelman 1980).

\section{Kelp deforestation}

Widespread kelp deforestation can result from disease, herbivory, and physiological stress or interactions among those processes. At lower latitude kelp forests (usually less than $40^{\circ}$ ), periodic deforestations results from oceanographic anomalies in temperature, salinity or nutrients that either kill kelps directly or trigger diseases that become lethal to physiologically-stressed plants. At mid-latitudes (about $40-60^{\circ}$ ), herbivory by sea urchins is the most common and most important agent of kelp deforestation. Latitudinal differences in patterns and processes shaping kelp forests have resulted in different researchers working in the same kelp forest system but reaching different conclusions (Foster 1990). Here we address the geography of kelp deforestation patterns and processes.

Kelp-free patches have probably always occurred at some scale but those created by physical factors tend to be relatively small and short-lived. The oldest term for algal deforestation is the Japanese word isoyake, which means 'rock burning' (D. Fujita, personal communication 2002). The word was coined by Yendo $(1902,1903)$ to describe algal deforestation in coastal zones of central Japan where the algal decline was thought to have resulted from salinity anomalies (Yendo 1903, 1914) rather than grazing, because herbivorous 
sea urchins were rare. The isoyake killed all foliacious algae first and then all encrusting coralline algae; the algae recovered several years later (Yendo 1914). Other mass mortalities of Ecklonia- and Eisenia-dominated kelp forests resulted from incursions of the Kuroshio Current along the central Japan coast (D. Fujita, personal communication 2002). On Honshu Island, near the southern limit of Japanese kelp, anomalous incursions of the warm Tsushima Current periodically create isoyake conditions. Such oceanographically-induced kelp deforestations are usually short-lived and reversible, as was the original isoyake case in Japan (Yendo 1914).

Kelp deforestations also result from El Niño events. Strong El Niños halt coastal upwelling of nutrient-rich water and cause surface waters to warm (Dayton et al. 1999). These anomalies in California caused patchy deforestation followed by rapid recovery (Tegner \& Dayton 1987; Tegner et al. 1997). Such physiological stresses are likely to be more common toward the low latitude limits of kelp ranges. For example, the northern limit of three species of brown algae in northern Chile shifted south toward higher latitudes following the El Niño event of 1982-1983 (Peters \& Breeman 1993). Such stresses may make kelps more susceptible to disease. Low-latitude kelps in northern New Zealand have succumbed to a disease that may have resulted from physiological stress (Cole \& Babcock 1996; Cole \& Syms 1999).

Within mid-latitudes (roughly between $40^{\circ}$ and $60^{\circ}$ latitude) where kelp development is less likely to be limited by physical processes such as temperature, nutrients and light, deforestation most often results from sea urchin herbivory (Table 2; Leighton et al. 1966; Lawrence 1975; Duggins 1980; Himmelman 1980; Dayton 1985a,b; Estes \& Duggins 1995; Mann 2000). This is most evident in the Northern Hemisphere where the most widespread and long-lasting herbivore-induced kelp deforestations have resulted from sea urchin grazing (Table 3). These primarily Laminaria-dominated kelp forests (Fig. 1) have been reduced in historical times to coralline-dominated 'urchin barrens' in the Aleutian Islands of Alaska, the Gulf of Maine, Canadian Maritimes (to Newfoundland; Himmelman 1980), northern Japan (Hokkaido Island), Iceland and northern Norway (Table 3). South of those regions, forests either remain intact, such as in southern California (Dayton et al. 1984), or are deforested patchily in relatively few regions, such as in southern Norway (Sivertsen 1997), Ireland (Kitching \& Thain 1983), the UK (Kain 1975) and southern Japan (Honshu Island; Fujita 1998).

Kelp deforestation from sea urchins is less common in the Southern Hemisphere (Table 3). In Chile, extensive sea urchin deforestation at mid latitudes $\left(54^{\circ}-46^{\circ} \mathrm{S}\right)$ was reported by Dayton (1985b). However that study and others (Table 3 ) report only patchy deforestation in north central Chile (i.e. $32^{\circ} \mathrm{S}$ latitude; Ojeda \& Santelices 1984). Dense Macrocystis forests with few urchins have been described in the southernmost regions of South America (around $55^{\circ} \mathrm{S}$ latitude) in Chile (Castilla \& Moreno 1982; Santelices \& Ojeda 1984b) and Argentina (Dayton 1985b). While south- ernmost Chile has four sea urchin species (Loxechinus albus, Pseudechinus magellanicus, Arbacia dufresnei and Austrocidaris canaliculata), they subsist on drift kelp and rarely graze attached Macrocystis plants. Along the east coast of southernmost South America to the northern limit of kelps in Argentina ( $42^{\circ} \mathrm{S}$ latitude; Barrales \& Lobban 1975), the sea urchin, Arbacia dufresnei is the dominant herbivore but its grazing impact on kelp forests is minimal (Barrales \& Lobban 1975).

Elsewhere in the Southern Hemisphere, sea urchin deforestation is patchy or restricted to particular depth zones. In New Zealand, a band of sea urchin 'barrens' exists at depths of around $10 \mathrm{~m}$ (Choat \& Schiel 1982). Urchins there may be prevented by wave turbulence from grazing shallower zones. In south Australia, kelp deforestation is restricted to regions of high spatial heterogeneity that provide shelters for nocturnally grazing sea urchins (Andrew 1993). Recent kelp deforestation in Tasmania resulted from newly established high-density sea urchin populations (C. Johnson, personal communication 2002). In South Africa, sea urchins alone do not overgraze kelp forests (Velimirov et al. 1977) but grazerinduced phase shifts can occur from a diversity of herbivores (G.M. Branch, personal communication 2002).

Whatever regulates sea urchin abundances or their grazing behaviour often controls the distribution and abundance of kelp forests (Tables $2 \& 3$ ). Predators are commonly strong interactors (sensu Paine 1980) and as such are the single most important agent controlling sea urchin populations (Cowen 1983; Duggins 1983; Tegner \& Levin 1983; Estes \& Duggins 1995; Sala et al. 1998; Steneck 1998). When sea urchin predators become the focus of intense and unsustainable fishing that extirpates them, hyperabundances of the sea urchins and kelp deforestation often result (Lawrence 1975; Estes \& Duggins 1995; Steneck 1998). Exceptions to this pattern are found at lower latitudes, where diverse guilds of kelp forest herbivores and predators compensate for the loss of a single predator species. Sea urchin abundance can also be influenced by other factors such as disease (Table 2; Fujita 1998; Scheibling et al. 1999), turbulence (Choat \& Schiel 1982) and storms (Dayton 1985a; Ebeling et al. 1985) that can locally or periodically reduce sea urchin abundance and thus control kelp forest development indirectly (Foster \& Schiel 1985).

Widespread, long-lasting kelp deforestation from sea urchins may be a relatively recent phenomenon. In the Aleutians of Alaska, the transition may have occurred early in the 20th century (Estes \& Duggins 1995). In Japan, fisherfolk observed deforestation and patches of corallines first in the early 1930s (Fujita 1987; 1998), although sea urchins were not mentioned at that time. Later, growing sea-urchingrazed coralline patches were reported during the 1950 s to 1960s (Ohmi 1951; Fujita 1998). In California during the 1960 s, the term 'barrens' was coined to describe sea urchininduced kelp deforestation (Leighton et al. 1966). In the North Atlantic, the first gaps in kelp forests were reported in the 1960s for the Gulf of Maine (Lamb \& Zimmerman 1964), Nova Scotia (Edelstein et al. 1969; Breen \& Mann 1976), 
Table 2 Present impacts on kelp forest ecosystems scored along a continuum: non-existent (blank), minimal (1), modest (2), great, but local (3), both great and widespread (4). The sign of the impact index number reflects whether impact tends to reduce kelp forests ( - ) or cause them to increase $(+)$. No sign indicates the impact can be positive or negative (for example, nutrient cycles).

\begin{tabular}{|c|c|c|c|c|}
\hline Potential impacts & $\begin{array}{l}\text { Direct human } \\
\text { impacts }\end{array}$ & $\begin{array}{l}\text { North-mest Atlantic } \\
\text { impacts }\end{array}$ & $\begin{array}{l}\text { Aleutians, Alaska } \\
\text { impacts }\end{array}$ & $\begin{array}{l}\text { Southern California } \\
\text { impact }\end{array}$ \\
\hline Direct kelp harvest & Yes & & & -1 (Dayton et al. 1998) \\
\hline Herbivory: sea urchin grazing & & -4 (Steneck 1997) & $\begin{array}{l}-4 \text { (Estes \& Duggins } \\
1995)\end{array}$ & $\begin{array}{l}-3 \text { (Leighton et al. } \\
\text { 1966) }\end{array}$ \\
\hline Fishing on sea urchins & Yes & $\begin{array}{l}+4 \text {, Maine, USA } \\
\text { (Steneck 1997) }\end{array}$ & & $\begin{array}{l}+1 \text { (Tegner \& Dayton } \\
\quad 1991)\end{array}$ \\
\hline Disease & & $\begin{array}{l}+4 \text {, Nova Scotia, Canada } \\
\text { (Scheibling 1986; } \\
\text { Steneck 1997; } \\
\text { Scheibling } \text { et al. } 1999 ; \\
\text { Levin } \text { et al. } 2003 \text { ) }\end{array}$ & $\begin{array}{l}+2 \text { (Pearse \& Hines } \\
\text { 1979) }\end{array}$ & \\
\hline Storms & & & & $\begin{array}{l}+2 \text { (Harris et al. } 1984 ; \\
\quad \text { Harrold \& Reed 1985) }\end{array}$ \\
\hline Herbivory: other grazers & & $\begin{array}{l}-2, \text { Lacuna snails (Johnson } \\
\quad \text { \& Mann 1986) }\end{array}$ & $\begin{array}{l}-1, \text { Steller's sea cow } \\
\text { (Domning 1972) }\end{array}$ & $\begin{array}{l}-1 \text {, Steller's sea cow, } \\
\text { abalone (Tegner \& } \\
\text { Levin 1983; Dayton } \\
\text { et al. 1999) }\end{array}$ \\
\hline \multicolumn{5}{|l|}{ Predation on sea urchins } \\
\hline Reduced (fishing pressure) & Yes & -4, cod (Steneck 1997) & $\begin{array}{l}-4 \text {, sea otter (Estes \& } \\
\text { Duggins 1995; Dayton } \\
\text { et al. 1998) }\end{array}$ & $\begin{array}{l}-3 \text {, sheephead fish } \\
\text { (Cowen 1983; Dayton } \\
\text { et al. 1998) }\end{array}$ \\
\hline Increased (predator switching) & & $\begin{array}{l}+2, \text { crabs }(\text { Tegner } \& \\
\quad \text { Levin } 1983)\end{array}$ & $\begin{array}{l}+3 \text {, killer whales (Estes } \\
\quad \text { et al. } 1998)\end{array}$ & \\
\hline Competition from non-native species & Yes & & & \\
\hline Epiphytes & Yes & $\begin{array}{c}\text {-2, bryzoan (Scheibling } \\
\text { 1986; Scheibling } \text { et al. } \\
\text { 1999; Levin } \text { et al. } 2003 \text { ) }\end{array}$ & & \\
\hline Exploitative competition & Yes & $\begin{array}{l}\text {-2, Codium sp. (Lambert } \\
\text { et al. 1992; Steneck \& } \\
\text { Carlton 2001) }\end{array}$ & & $\begin{array}{l}-1, \text { Sargassum sp. } \\
\quad \text { (Dayton et al. 1998) }\end{array}$ \\
\hline Pollution & Yes & & & \\
\hline Eutrophication & Yes & & & -1 (Tegner et al. 1995) \\
\hline Sedimentation & Yes & & & -1 (Tegner et al. 1995) \\
\hline Oil spills & Yes & & $\begin{array}{l}-1 \text { (Dean \& Jewett } \\
\text { 2001) }\end{array}$ & $\begin{array}{l}-1 \text { (Dayton \& Tegner } \\
\text { 1990) }\end{array}$ \\
\hline \multicolumn{5}{|l|}{ Climate change } \\
\hline El Niño & & & & $\begin{array}{l}\text {-3 (Tegner \& Dayton } \\
\text { 1987, 1991; Dayton } \\
\text { et al. 1998) }\end{array}$ \\
\hline La Niña & & & & +2(Dayton et al. 1998) \\
\hline Global warming & Yes & 1 & & -1 (Dayton et al. 1998) \\
\hline Storm frequency and intensity & & -3 (Witman 1987) & $\begin{array}{c}-2 \text { (Ebeling et al. } 1985 ; \\
\text { Tegner et al. 1996a) }\end{array}$ & $\begin{array}{l}\text {-3 (Pearse \& Hines } \\
\text { 1979; Ebeling et al. } \\
\text { 1985; Tegner } \text { et al. } \\
\text { 1996a; Dayton } \text { et al. } \\
\text { 1998) }\end{array}$ \\
\hline \multicolumn{5}{|l|}{ Season cycles } \\
\hline Nutrient availability & & $\begin{array}{c}1 \text { (Chapman \& Craigie } \\
\text { 1977; Gerard 1997) }\end{array}$ & & $\begin{array}{l}2 \text { (Gerard 1982; } \\
\quad \text { Zimmerman \& } \\
\quad \text { Kremer 1984, 1986) }\end{array}$ \\
\hline
\end{tabular}

Ireland (Ebling et al. 1966), and the UK (Jones \& Kain 1967). Gaps in kelp forests in the western North Atlantic coalesced and expanded during the 1970s and 1980s in Nova Scotia (Mann 1977) and the Gulf of Maine (Steneck 1997).
Expansive coralline 'barrens' existed in Newfoundland in the late 1960s (Himmelman 1980) and possibly were present there earlier (Hooper 1980). In the eastern North Atlantic, widespread urchin-induced deforestation was first observed 
Table 3 Comparison of subtidal kelp forest ecosystems of the world. Numbers in parentheses denote number of ecologically important species in subtidal kelp forests for specified taxa.

\begin{tabular}{|c|c|c|c|c|c|c|c|c|c|}
\hline \multicolumn{5}{|c|}{ Kelps and their controlling agents } & \multicolumn{5}{|c|}{ Spatial and temporal scale of deforestation } \\
\hline $\begin{array}{l}\text { Site and } \\
\text { latitude }\end{array}$ & $\begin{array}{l}\text { Dominant } \\
\text { kelps }\end{array}$ & $\begin{array}{l}\text { Deforesting } \\
\text { herbivores }\end{array}$ & $\begin{array}{l}\text { Predators or } \\
\text { diseases of kelp } \\
\text { herbivores }\end{array}$ & $\begin{array}{l}\text { Drift kelp, } \\
\text { disease, } \\
\text { oceanography }\end{array}$ & $\begin{array}{l}\text { Regional } \\
\text { distribution }\end{array}$ & $\begin{array}{l}\text { Depth } \\
\text { range }\end{array}$ & $\begin{array}{l}\text { Local } \\
\text { distribution }\end{array}$ & $\begin{array}{l}\text { Duration } \\
\text { deforested }\end{array}$ & References \\
\hline \multicolumn{10}{|c|}{ Western North Atlantic } \\
\hline $\begin{array}{l}\text { Nova Scotia } \\
43-45^{\circ} \mathrm{N}\end{array}$ & $\begin{array}{c}\text { Laminaria }(1), \\
\text { Agarum }(1)\end{array}$ & Echinoid (1) & $\begin{array}{l}\text { Fishes (2), } \\
\quad \text { urchin disease }\end{array}$ & $\begin{array}{l}\text { Drift, } \\
\text { disease }\end{array}$ & Widespread & Broad & Homogenous & Decades & $\begin{array}{l}\text { Tables 1, 2, 4; } \\
\quad \text { Fig. } 5\end{array}$ \\
\hline $\begin{array}{l}\text { Maine } \\
43-44^{\circ} \mathrm{N}\end{array}$ & $\begin{array}{c}\text { Laminaria }(1), \\
\text { Agarum }(1)\end{array}$ & Echinoid (1) & $\begin{array}{l}\text { Fishes }(2) \\
\quad(\text { crabs })\end{array}$ & & Widespread & Broad & Homogenous & $>$ Decades & $\begin{array}{l}\text { Tables 1, 2, 4; } \\
\quad \text { Fig. } 4\end{array}$ \\
\hline \multicolumn{10}{|c|}{ Eastern North Atlantic } \\
\hline $\begin{array}{l}\text { North Iceland } \\
65^{\circ} \mathrm{N}\end{array}$ & Laminaria (1) & Echinoid (1) & $?$ & & Widespread & Broad & Homogenous & $?$ & $\begin{array}{l}\text { Hjorleifsson } \\
\text { et al. }(1995) ; \\
\text { W. H. Adey } \\
\text { personal } \\
\text { communication } \\
\text { on }(2001)\end{array}$ \\
\hline $\begin{array}{l}\text { North Norway } \\
65-71^{\circ} \mathrm{N}\end{array}$ & Laminaria (1) & Echinoid (2) & Seabirds & & Widespread & Broad & Homogenous & Decades & $\begin{array}{l}\text { Hagen (1983); } \\
\text { Bustnes et al. } \\
\text { (1995); } \\
\text { Sivertsen } \\
\text { (1997) }\end{array}$ \\
\hline $\begin{array}{l}\text { South Norway } \\
55-64^{\circ} \mathrm{N}\end{array}$ & Laminaria (1) & Echinoid (1) & $?$ & & Restricted & Broad & Patchy & Decades & Sivertsen (1997) \\
\hline $\begin{array}{l}\text { Britain and } \\
\text { Ireland } \\
52-55^{\circ} \mathrm{N}\end{array}$ & Laminaria (3) & Echinoids (2) & Crabs & & Restricted & Broad & Patchy & & $\begin{array}{l}\text { Kitching \& } \\
\text { Ebling (1961); } \\
\text { Ebling et al. } \\
\text { (1966); Kain } \\
\text { (1975) }\end{array}$ \\
\hline \multicolumn{10}{|l|}{ East North Pacific } \\
\hline $\begin{array}{l}\text { Alaska } \\
\text { (Aleutians) } \\
50-55^{\circ} \mathrm{N}\end{array}$ & $\begin{array}{l}\text { Alaria (1), } \\
\quad \text { Laminaria (3), } \\
\text { Thalassiophyllum } \\
\text { (1), Agarum (1) }\end{array}$ & Echinoid (1) & Sea otter & & Widespread & Broad & Homogenous & $>$ Decades & $\begin{array}{l}\text { Tables 1, 2, 4; } \\
\quad \text { Fig. } 3\end{array}$ \\
\hline $\begin{array}{l}\text { Southern } \\
\text { California } \\
30-35^{\circ} \mathrm{N}\end{array}$ & $\begin{array}{l}\text { Macrocystis }(1), \\
\text { Laminaria }(1), \\
\text { Pterygophora }(1)\end{array}$ & $\begin{array}{l}\text { Echinoids (3), } \\
\text { gastropods } \\
\text { (8), fishes (2) }\end{array}$ & $\begin{array}{l}\text { Sea otter }(1), \\
\text { fish (1), } \\
\text { lobster (1) }\end{array}$ & $\begin{array}{l}\text { Drift, } \\
\text { Oceanographic } \\
\text { (ENSO) } \\
\text { events }\end{array}$ & Restricted & Broad & Patchy & $<$ Decade & $\begin{array}{l}\text { Tables } 1,2,4 ; \\
\text { Figs. } 6,7\end{array}$ \\
\hline \multicolumn{10}{|l|}{ West North Pacific } \\
\hline $\begin{array}{l}\text { North Japan } \\
\text { (SW Hokkaido) } \\
39-46^{\circ} \mathrm{N}\end{array}$ & Laminaria (2) & Echinoid (1-3) & $\begin{array}{l}\text { Crabs, urchin } \\
\text { disease }\end{array}$ & $\begin{array}{l}\text { Oceanographic } \\
\text { event }\end{array}$ & Widespread & Broad & Homogenous & Decades & $\begin{array}{l}\text { Fujita (1998); } \\
\text { D. Fujita, } \\
\text { personal } \\
\text { communication } \\
(2002)\end{array}$ \\
\hline $\begin{array}{l}\text { South Japan } \\
\text { (West Honshu) } \\
36-38^{\circ} \mathrm{N}\end{array}$ & $\begin{array}{l}\text { Undaria }(1), \\
\text { Eisenia }(1), \\
\text { Ecklonia }(1)\end{array}$ & $\begin{array}{l}\text { Echinoid (3), } \\
\text { fish (1) }\end{array}$ & $?$ & $\begin{array}{l}\text { Oceanographic } \\
\text { event }\end{array}$ & Restricted & Broad & Patchy & $?$ & $\begin{array}{l}\text { Fujita (1998); D. } \\
\text { Fujita, personal } \\
\text { communication } \\
\text { (2002) }\end{array}$ \\
\hline $\begin{array}{l}\text { East South Pacific } \\
\text { North Chile } \\
18^{\circ}-42^{\circ} \mathrm{S}\end{array}$ & $\begin{array}{l}\text { Lessonia (1), } \\
\qquad \text { Macrocystis }(1)\end{array}$ & $\begin{array}{l}\text { Echinoids (2), } \\
\text { fishes (1), } \\
\text { gastropods (2) }\end{array}$ & $\begin{array}{l}\text { Asteroids (3), } \\
\text { fishes (3) }\end{array}$ & Drift & Widespread & Shallow & Patchy & Decades & $\begin{array}{l}\text { Ojeda \& Santilices } \\
\text { (1984); Vasquez } \\
\text { (1993); Vasquez } \\
\text { \& Buschmann } \\
\text { (1997) }\end{array}$ \\
\hline $\begin{array}{l}\text { South Chile } \\
46^{\circ}-54^{\circ} \mathrm{S}\end{array}$ & $\begin{array}{c}\text { Macrocystis }(1) \\
\text { Lessonia }(2)\end{array}$ & $\begin{array}{l}\text { Echinoids (1), } \\
\text { gastropods (1) }\end{array}$ & Asteroids (1) & Drift & Restricted & Shallow & Homogenous & Decades & Dayton (1985b) \\
\hline $\begin{array}{l}\text { Southernmost } \\
\text { Chile } \\
55^{\circ} \mathrm{S}\end{array}$ & $\begin{array}{l}\text { Macrocystis }(1) \\
\text { Lessonia }(2)\end{array}$ & Echinoids (4) & Asteroids (1) & Drift & None & & & & $\begin{array}{l}\text { Castilla \& } \\
\text { Moreno (1982); } \\
\text { Santelices \& } \\
\text { Ojeda (1984b); } \\
\text { Vasquez et al. } \\
\text { (1984) }\end{array}$ \\
\hline $\begin{array}{l}\text { Argentina } \\
42^{\circ}-55^{\circ} \mathrm{S}\end{array}$ & $\begin{array}{c}\text { Macrocystis (1), } \\
\text { Lessonia (1) }\end{array}$ & Echinoid (1) & $?$ & $?$ & None & & & & $\begin{array}{l}\text { Barrales \& } \\
\quad \text { Lobban (1975) }\end{array}$ \\
\hline
\end{tabular}


Table 3 Continued

\begin{tabular}{|c|c|c|c|c|c|c|c|c|c|}
\hline \multicolumn{5}{|c|}{ Kelps and their controlling agents } & \multicolumn{5}{|c|}{ Spatial and temporal scale of deforestation } \\
\hline $\begin{array}{l}\text { Site and } \\
\text { latitude }\end{array}$ & $\begin{array}{l}\text { Dominant } \\
\text { kelps }\end{array}$ & $\begin{array}{l}\text { Deforesting } \\
\text { herbivores }\end{array}$ & $\begin{array}{l}\text { Predators or } \\
\text { diseases of kelp } \\
\text { herbivores }\end{array}$ & $\begin{array}{l}\text { Drift kelp, } \\
\text { disease, } \\
\text { oceanography }\end{array}$ & $\begin{array}{l}\text { Regional } \\
\text { distribution }\end{array}$ & $\begin{array}{l}\text { Depth } \\
\text { range }\end{array}$ & $\begin{array}{l}\text { Local } \\
\text { distribution }\end{array}$ & $\begin{array}{l}\text { Duration } \\
\text { deforested }\end{array}$ & References \\
\hline \multicolumn{10}{|l|}{ West South Pacific } \\
\hline $\begin{array}{l}\text { Australia } \\
\text { (New South } \\
\text { Wales) } \\
\sim 32^{\circ}-35^{\circ} \mathrm{S}\end{array}$ & Ecklonia $(1)$ & $\begin{array}{l}\text { Echinoids (1), } \\
\text { fishes (1) }\end{array}$ & Fishes (2) & & Widespread & $\begin{array}{l}\text { Moderately } \\
\text { deep }\end{array}$ & Patchy & $?$ & $\begin{array}{l}\text { Andrew (1993, } \\
\text { 1994); Andrew } \\
\text { \& Underwood } \\
\text { (1993); Andrew } \\
\text { \& O'Neill } \\
(2000)\end{array}$ \\
\hline $\begin{array}{l}\text { Australia } \\
\text { (Tasmania) } \\
\sim 43^{\circ} \mathrm{S}\end{array}$ & $\begin{array}{c}\text { Macrocystis (1), } \\
\text { Ecklonia (1) }\end{array}$ & Echinoid (1) & $\begin{array}{l}\text { Fish }(1), \\
\quad \text { lobster (1) }\end{array}$ & & Restricted & Broad & Homogenous & Years & $\begin{array}{l}\text { C. Johnson, } \\
\text { personal } \\
\text { communication } \\
(2002)\end{array}$ \\
\hline $\begin{array}{l}\text { New Zealand } \\
\text { (North Island) }\end{array}$ & $\begin{array}{l}\text { Ecklonia }(1), \\
\text { Lessonia }(1)\end{array}$ & $\begin{array}{l}\text { Echinoids (2), } \\
\text { gastropods (2) }\end{array}$ & $\begin{array}{l}\text { Fishes (?), } \\
\text { lobster (1) }\end{array}$ & Kelp disease & Widespread & Mid-depth & Homogenous & Decade & \\
\hline $34^{\circ}-37^{\circ} \mathrm{S}$ & & & & & & & & & $\begin{array}{l}\text { Andrew \& } \\
\text { Choat (1982); } \\
\text { Choat \& } \\
\text { Schiel (1982); } \\
\text { Choat \& } \\
\text { Ayling (1987); } \\
\text { Schiel (1990); } \\
\text { Cole \& } \\
\text { Babcock (1996); } \\
\text { Babcock et al. } \\
\text { (1999); Cole } \\
\text { \& Syms } \\
\text { (1999) }\end{array}$ \\
\hline $\begin{array}{l}\text { New Zealand } \\
\text { (South Island) } \\
41^{\circ}-47^{\circ} \mathrm{S}\end{array}$ & $\begin{array}{l}\text { Ecklonia }(1), \\
\quad \text { Lessonia }(1), \\
\quad \text { Macrocystis }(1)\end{array}$ & Echinoids (1) & $?$ & & Restricted & Broad & Patchy & $?$ & $\begin{array}{l}\text { Schiel 1990; } \\
\text { Schiel et al. } \\
\quad(1995)\end{array}$ \\
\hline \multicolumn{10}{|l|}{ East South Atlantic } \\
\hline $\begin{array}{l}\text { South Africa } \\
\sim 30^{\circ}-35^{\circ} \mathrm{S}\end{array}$ & $\begin{array}{l}\text { Ecklonia }(1), \\
\quad \text { Laminaria }(1), \\
\quad \text { Macrocystis }(1)\end{array}$ & $\begin{array}{l}\text { Echinoids (1), } \\
\quad \text { gastropods (1) }\end{array}$ & $\begin{array}{l}\text { Lobster (1), } \\
\text { fish (?) }\end{array}$ & & Widespread & Deep only & Patchy & $?$ & $\begin{array}{l}\text { Anderson et al. } \\
\qquad \begin{array}{l}\text { (1997); G. } \\
\text { Branch, } \\
\text { personal } \\
\text { communication } \\
(2001)\end{array}\end{array}$ \\
\hline \multicolumn{10}{|l|}{ East Indian Ocean } \\
\hline $\begin{array}{l}\text { Western } \\
\text { Australia } \\
28^{\circ} \mathrm{S}\end{array}$ & Ecklonia (1) & $\begin{array}{l}\text { Oceanography } \\
\text { (high } \\
\text { temperature } \\
\text { and low } \\
\text { nutrients) }\end{array}$ & & & & & & & $\begin{array}{l}\text { Hatcher et al. } \\
\quad(1987)\end{array}$ \\
\hline
\end{tabular}

in northern Norway in the early 1980s (Hagen 1983; Sivertsen 1997) and in Iceland in the early 1990s (Hjorleifsson et al. 1995). By the mid-1970s, sea urchins were viewed as the major cause of kelp deforestation (Lawrence 1975) such that by the mid-1980s conferences were held to discuss (among other things) how sea urchins could be eradicated (Pringle et al. 1980; Bernstein \& Welsford 1982). Sea urchin-induced kelp deforestation was widely reported in mid-latitudes of the Northern Hemisphere from $40^{\circ}-60^{\circ} \mathrm{N}$ (higher in the eastern North Atlantic due to the Gulf Stream) during the 1960s and 1980s. At the time, some researchers openly wondered if kelp deforestation was an irreversible degradation' (Mann 1977).

\section{LONG TERM TRENDS: THREE NORTH AMERICAN CASE STUDIES}

Kelp forests live in a balance between forces contributing to their development and deforestation. The geography of both physical and biological forcing functions for kelp forest development and persistence is complex. We know that many kelp forests look and behave very differently today from their historical counterparts. Many of these differences are due, directly or indirectly to human perturbation of physical or biological components of kelp ecosystems. Some of the most impressive changes result from trophic cascades, largely through the functional elimination of apex predators. These play out over a period of decades, centuries or longer. Here we illustrate this point with long-term historical 
chronologies, some known and others inferred, from Alaska, the western North Atlantic, and southern California. These three systems are among the best studied in the world and the only ones we know for which archaeological data exist.

\section{Alaska}

The kelp forest ecosystems of the eastern North Pacific likely arose during the last $20-40$ million years with the evolution of kelps, strongylocentrotid sea urchins, sea otters, and the now extinct Steller's sea cow (Estes et al. 1989). Kelp forests probably dominated ice-free coasts throughout this region since the last glacial period (Table 4). During the Pleistocene, sea cows ranged from Japan, throughout the North Pacific to at least Monterey Bay in California (Jones 1967). They may have been extirpated from most of their range by aboriginal hunting at the end of the Pleistocene and early Holocene, because they survived thousands of years longer in the Commander Islands, a region that was not peopled until the time of European contact in 1741 (Estes et al. 1989). European fur traders killed the last sea cow 27 years later. The impact of sea cows on kelp forests is unknown. However they had no teeth, probably were unable to dive and thus were most likely a trophic specialist of canopy kelps (Domning 1972; Clementz 2002). Kelp canopies are remarkably resilient to cropping of their most distal fronds and currently, in California, they support a multi-million dollar industry of canopy-cropping factory ships that sustainably harvest kelp for their valuable alginates. If harvesting factory ships do little permanent damage to kelp forests (Tegner \& Dayton 2000), it is unlikely that sea cow grazing of canopies deforested kelp beds.

It is possible that the concentration of food associated with highly productive North Pacific kelp forests attracted early maritime people and facilitated some of the earliest migration of people from Eurasia to the Americas. It is now apparent that anatomically modern humans had colonized the Ryuku Islands south of Japan between 35000 and 25000 years ago and that boats were in use along the Japanese coast by about 25000 years ago (Erlandson 2001). The readily-available shellfish, finfish, and marine birds and mammals may have allowed human populations to become established, proliferate and grow well before landward migration and the eventual development of agriculture began. Furthermore, climatic conditions along the land-sea margin were benign compared with more inland areas. It is possible that the whaling tradition of indigenous people of the North Pacific began with the overharvest of the predator-naive and defenceless Steller's sea cow, focusing thereafter on cetaceans that were more difficult to harvest, once the sea cows were extirpated (Domning 1972). Currently, the earliest evidence for human occupation of the southern Alaskan coast (Aleutians to south-east Alaska) dates to between 9000 and 10000 years ago, although rising post-glacial sea levels may well have submerged earlier sites.

Alaskan kelp forests are likely to have been well developed before human contact because sea otter predation on sea

Table 4 Spatial and temporal scale of change among three kelp forest ecosystems in North America.

\begin{tabular}{|c|c|c|c|}
\hline Event & North-west Atlantic & West Aleutian islands & Southern California \\
\hline $\begin{array}{l}\text { Pristine state (prior to } \\
\text { human contact) }\end{array}$ & Kelp forest & Kelp forest & Kelp forest \\
\hline Scale (patch size) & $\begin{array}{l}100-500 \mathrm{~km}^{2} \text { (Johnson \& Mann } \\
1988)\end{array}$ & $200-1000 \mathrm{~km}^{2}$ (Estes et al. 1989) & $\begin{array}{l}<10 \mathrm{~km}^{2} \text { (Harrold \& Reed 1985; } \\
\quad \text { Tegner } \text { et al. 1996a) }\end{array}$ \\
\hline $\begin{array}{l}\text { First human } \\
\text { contact/occupation } \\
\text { (years before present) }\end{array}$ & 10000 (Bourque 1995) & 8000 (Simenstad et al. 1978) & $\begin{array}{l}12000-13000 \text { (Erlandson } \\
\quad \text { et al. 1996) }\end{array}$ \\
\hline $\begin{array}{l}\text { Marine organisms } \\
\text { present in diet } \\
\text { (years before present) }\end{array}$ & 5000 (Bourque 1996) & 4500 (Simenstad et al. 1978) & 11600 (Erlandson et al. 1996) \\
\hline $\begin{array}{l}\text { First known phase change } \\
\text { (years before present) }\end{array}$ & ?-40 (Adey 1964) & 2500 (Simenstad et al. 1978) & $\begin{array}{l}\text { 4000-6000 (Salls 1991, 1995; } \\
\text { Erlandson } \text { et al. 1996; } \\
\text { Erlandson \& Rick 2002) }\end{array}$ \\
\hline $\begin{array}{l}\text { First European } \\
\text { contact/exploitation } \\
\text { (years before present) }\end{array}$ & 460-400 (Steneck 1997) & 260 (Simenstad et al. 1978) & 460-200 (Simenstad et al. 1978) \\
\hline $\begin{array}{l}\text { Kelp bed re-establishment } \\
\text { rate (years) }\end{array}$ & $\begin{array}{l}\text { 0.5-4 (Johnson \& Mann 1988; } \\
\quad \text { Dayton et al. 1999) }\end{array}$ & 2 (Estes et al. 1989) & $\begin{array}{l}0.5 \text { (Harrold \& Reed 1985; } \\
\text { Tegner } \text { et al. 1996a) }\end{array}$ \\
\hline Alternate (kelp free) state & Coralline/urchin & Coralline/urchin & Coralline/urchin \\
\hline $\begin{array}{l}\text { Storms remove dominant } \\
\text { kelp }\end{array}$ & $\begin{array}{l}\text { Small scale removal (Johnson \& } \\
\quad \text { Mann 1988) }\end{array}$ & - & Large scale removal \\
\hline Recent apex predators & Crabs (Leland \& Steneck 2001) & Killer whales (Estes et al. 1998) & 一 \\
\hline Introduced competitors & $\begin{array}{l}\text { Bryozoan, Codium (Lambert } \\
\text { et al. 1992) }\end{array}$ & & \\
\hline
\end{tabular}


Figure 2 Timing of phase changes in community state of kelp forests of North America. Kelp with vertebrate predators, sea urchins without kelp and kelp without predators have been identified for some or all of the case study locations. Kelp forests are listed from the greatest number of trophic levels on the left to fewest trophic levels on the right. Case studies are listed from lowest species diversity in Maine to highest diversity in southern California. See text or Table 4 for references.

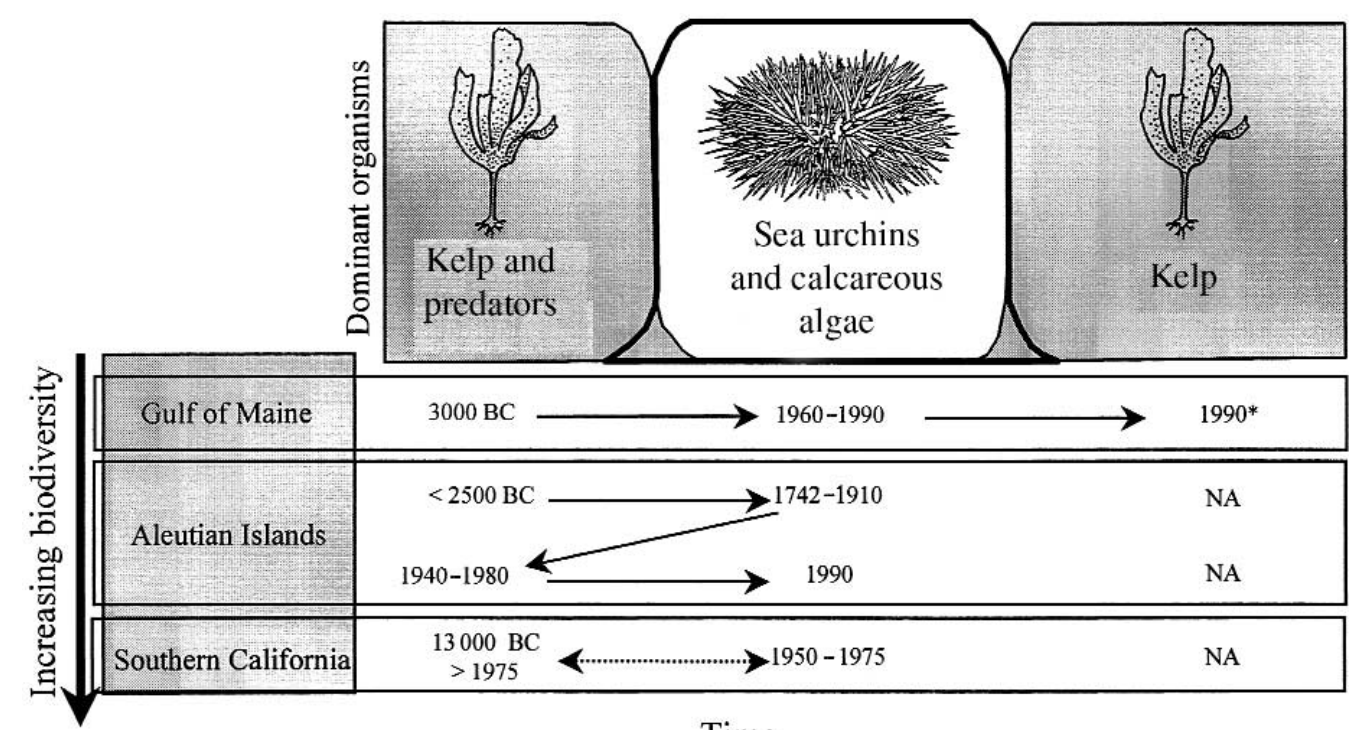

Time

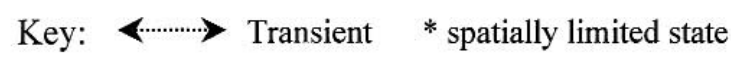

urchins prevented overgrazing on kelp (Simenstad et al. 1978; Estes et al. 1998; left side Fig. 2). Aboriginal Aleuts greatly diminished sea otters beginning around $2500 \mathrm{yr}$ BP with a corresponding increase in the size of sea urchins (Simenstad et al. 1978). European and North American fur traders subsequently hunted the remaining otters to the brink of extinction in the 1700 and 1800 s, causing the collapse of kelp forests as they were grazed away by sea urchins released from sea otter predation (middle of Fig. 2 and Fig. 3). Legal protection of sea otters in the 20th century reversed their decline and the resultant trophic cascade (Fig. 3), but kelp

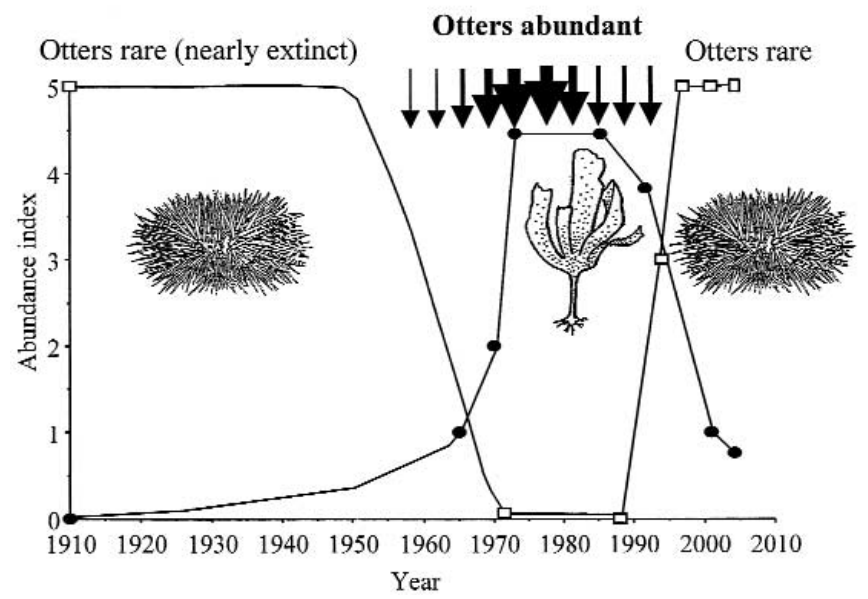

Figure 3 Temporal trends in kelp forests, predators and sea urchins of Amchitka, Alaska. Abundances estimated from several studies (see text) and Estes and Duggins (1995). Arrows indicate the timing of change in major community-changing forcing functions. Width of arrowheads indicates magnitude of the forcing function's impact. forests have disappeared again over vast areas of the southwest Alaska coast as sea otter populations have fallen prey to killer whales (Estes et al. 1998). The whales apparently shifted their diet to sea otters from seals and sea lions, after the latter populations declined significantly. The pinniped declines are likely to have been caused by changes in the open ocean. Whether these changes were natural or anthropogenic remains uncertain.

\section{Western North Atlantic}

Kelps and sea urchins in the Gulf of Maine were derived from the North Pacific by way of the transarctic interchange (Durham \& MacNeil 1967; Vermeij 2001). Sea otters did not make this journey. On arriving in the north-western Atlantic, however, sea urchins encountered functionally-similar predators, namely Atlantic cod and other large groundfish, which maintained the kelp-dominated state via a trophic cascade. These predatory fishes have been present in the Gulf of Maine archaeological record for at least 5000 years (Bourque 1995; Steneck 1997).

Indigenous fishers exploited cod by hook and line for thousands of years (Bourque 1995, 2001; Steneck 1997). They maintained a varied diet of marine organisms such as cod, other fish, oysters and clams, as well as terrestrial animals such as deer and sea mink, the latter now extinct (Bourque 1995). When the first Europeans explored the Gulf of Maine, the abundance of large fish impressed them (Rosier 1605). Vespucci marked the western North Atlantic coast on his 1526 map of the New World Bacallaos, which is Portuguese for 'land of the codfish'. In 1602, Bartholomew Gosnold named Cape Cod for the myriad fish that 'vexed' his ship. By all accounts, cod and other large predatory fish were stable components of coastal zones throughout the western 
North Atlantic (Steneck 1997). Further, in rare offshore habitats where large predatory fishes still persist, urchins are rare, kelp is abundant (Vadas \& Steneck 1988, 1995) and attack rates on tethered sea urchins are high (Vadas \& Steneck 1995). Significantly, very small urchins (a few millimetres in diameter) were found at this site indicating that they can recruit but do not, probably because of high fish predation rates (Vadas \& Steneck 1995).

We surmise that kelp forests dominated the benthos while predatory finfish were abundant in coastal zones through at least the 1930s. The earliest reports of algae support that supposition. Hervey (1881) described all three dominant kelp species (Table 1 ) as being 'very abundant from Greenland to Cape Cod' and often 'washed ashore in great numbers'. Windrows of kelp detritus are good indicators of a kelpforested state (Novaczek \& McLachlan 1986), there was no mention of expansive patches of coralline algae at that time and the earliest scientific study in the region (Johnson \& Skutch 1928) reported that kelps were the 'most characteristic plant in the midlevels of the sublittoral zone.' Similarly, Nova Scotia was described as kelp-dominated in the early 1950s (MacFarlane 1952).

Extensive fishing grounds for cod and other predatory fishes were first mapped for coastal zones in Maine in the 1880 s and then again in the 1920 s, with remarkably little change in areal extent or location (Steneck 1997). Cod stocks persisted until mechanized fishing technology and on-board refrigeration allowed spawning aggregations of cod to be targeted in the 1930s (Rich 1929; Conkling \& Ames 1996). This set off a rapid decline in the numbers and body size of coastal cod in the Gulf of Maine (Steneck 1997; Jackson et al. 2001). Data from 5000 year old Indian middens, and from fisheries over the past century document a relatively recent but rapid decline in the average cod body-size, coincident with their extirpations from coastal zones (Jackson et al. 2001; Steneck \& Carlton 2001). Dominant fish predators in the coastal zone were replaced by small, commercially less important species such as sculpins (Steneck 1997). Today, large predatory finfishes remain functionally absent from coastal regions of the western North Atlantic (Steneck 1997).

Predatory fishes consume and control the distribution and abundance of sea urchins (Keats et al. 1986; Vadas \& Steneck 1995). The extirpation of coastal cod and other fishes by the 1940s in the Gulf of Maine resulted in functional loss of apex predators, which fundamentally altered coastal food webs as lobsters, crabs and sea urchins all increased in abundance (Steneck 1997). In the 1960s, scuba diving allowed coastal ecosystems to be observed and described in situ for the first time. The coastal Gulf of Maine was described then as a mosaic of kelp forests (Lamb \& Zimmerman 1964) and widely spaced 'barren' patches of sea urchins and coralline algae (Adey 1964; W.H. Adey, personal communication 2001; Fig. 4). Similar patches were described a decade later in Nova Scotia (Breen \& Mann 1976; Fig. 5). Over the next two decades, sea urchin abundances increased throughout the Gulf of Maine, kelp forests declined and coralline barrens

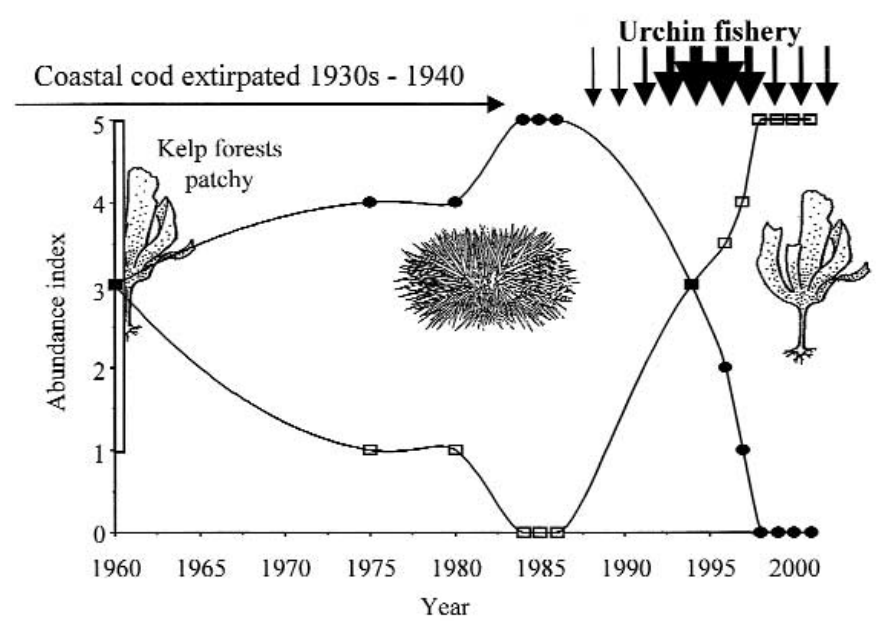

Figure 4 Temporal trends in kelp forests and sea urchins in the Gulf of Maine in the western North Atlantic. Width of arrowheads indicates the magnitude of the forcing function's impact.

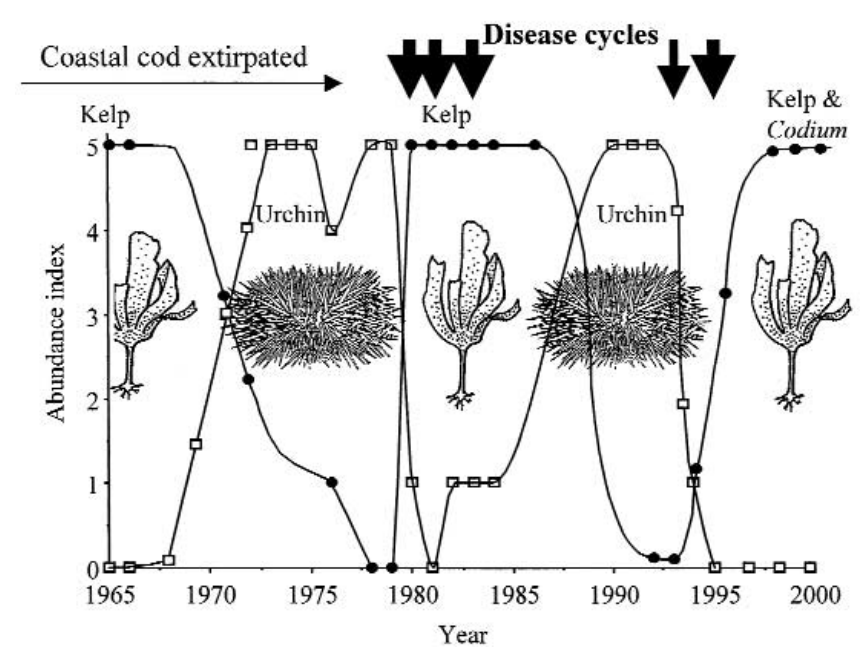

Figure 5 Temporal trends in kelp forests and sea urchins of Nova Scotia. Abundances estimated from Edelstein et al. (1969), Breen and Mann (1976), Warton and Mann (1981), Scheibling and Stephenson (1984), Scheibling (1986) and Johnson and Mann (1988). Width of arrowheads indicates the magnitude of the forcing function's impact.

grew and coalesced (Steneck 1997; Fig. 2). From the mid 1980s to the early 1990s, kelp forests reached an all-time low in their distribution and abundance throughout the region (Steneck 1997). Similar developments were observed in Nova Scotia, except that the system there was punctuated with disease-induced mass mortality of urchins causing it to rapidly oscillate between forested and deforested states (Fig. 6, discussed below). Arguably, the conditions for high densities of sea urchins that repeatedly denuded coastal zones of eastern Nova Scotia were only possible after the system became functionally free of apex-predators (middle and right sides of Fig. 2). 


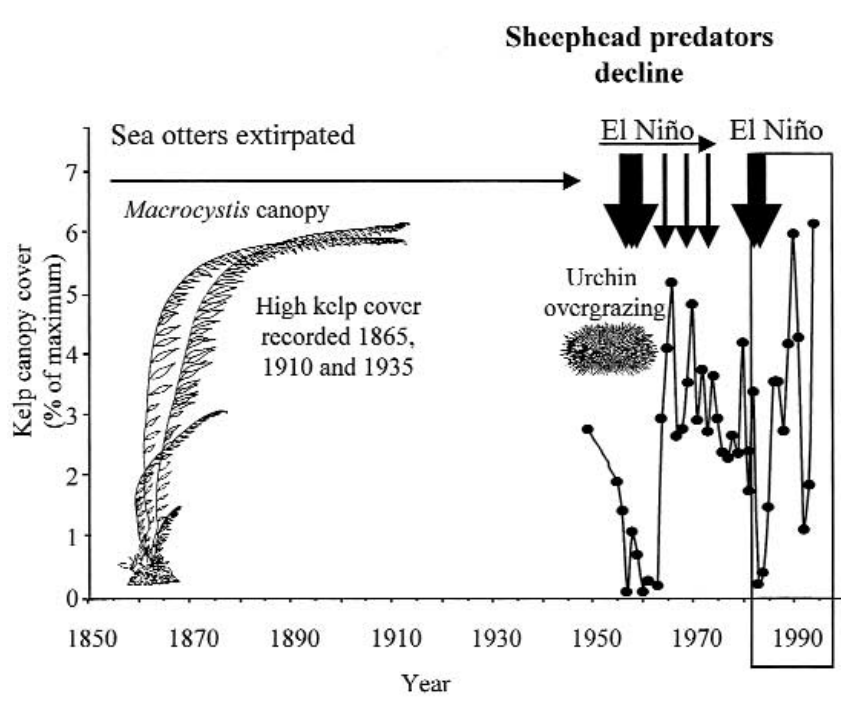

Figure 6 Temporal trends in kelp forests of Point Loma California. Abundance estimates summarized in Leighton et al. (1966), Tegner et al. (1996a), McGowan et al. (1998) and Tegner and Dayton (2000). Width of arrowheads indicates the magnitude of the forcing function's impact. The boxed area on the right of the figure indicates a period of high resolution subtidal data (see Fig. 3).

In 1987, a fishing industry developed to harvest Maine's sea urchins for their highly valued roe. This caused a remarkably rapid decline in urchin distribution and abundance (Steneck 1997; Vavrinec 2003). In response, kelp forests recovered, but were now without a functional herbivore trophic level (right side of Fig. 2). As a result, primary production was enhanced, and the revegetated habitat also increased the recruitment potential for some fish (Levin 1991, 1994) and crabs (McNaught 1999). Newly-settled crabs (Cancer spp.) survived in high numbers and became micropredators of settling sea urchins. The micropredator impact of crabs on urchins is striking because they can consume entire cohorts of settled sea urchins over large areas. Areas with rates of urchin settlement exceeding 10000 individuals $\mathrm{m}^{-2}$ had no survivors a year later due to crab micropredation (McNaught 1999). The phase change to macroalgae in the absence of large predatory finfish allowed large populations of adult-sized crabs to accumulate. Experiments to reintroduce adult sea urchins to population densities historically maintained prior to the sea urchin fishery resulted in attacks by swarms of large crabs (Leland 2002). In 2000, all 24000 introduced urchins were consumed within two months. Similar patterns were observed in 2001 on a second attempt to reintroduce adult urchins to the region (Leland 2002). Thus a new 'apex' predator has emerged in coastal zones of the Gulf of Maine with functionally the same impact of vertebrate apex predators. Fished-down coastal food webs in the western North Atlantic resulted in an herbivore-free alternate stable state dominated for the time being by kelp.

\section{California}

The highly diverse food web of southern California kelp forests historically included several herbivorous species of sea urchins, small crustaceans, abalone and other snails as well as their predators including sea otters, spiny lobsters and large sheephead labrid fish (Table 1). At the time of early European contacts (AD 1542-1769), the kelp-laden and protected coastlines of the southern California Bight supported one of the highest concentrations of hunter-gatherers in human history. Archaeological evidence shows that maritime Indians colonized California's northern Channel Islands at least 12000-13000 years ago (Erlandson et al. 1996; left side of Fig. 2; Table 4). These early maritime people had seaworthy boats and by 10000 years ago developed the first hook and line fishery in the Americas (Rick et al. 2001). They relied heavily on marine resources associated with kelp forests such as abalone shellfish, sheephead and marine mammals (Erlandson et al. 1996). Human settlement proliferated through the Holocene and later Indian peoples may have exploited kelp forest predators and herbivores with sufficient intensity to cause localized phase-shifts. In San Clemente Island middens, the size of sheephead bones decreased as prehistoric human populations increased with increased fishing intensity and a later overlying stratum was composed almost entirely of purple sea urchin remains (Salls 1991, 1995). Similar urchin 'lenses' have been noted at several San Miguel Island sites, all of which, so far, date to the last 3500-4500 years. This suggests that Indians may have created local sea-urchin barrens by hunting such sea urchin predators as sea otters and sheephead (Erlandson et al. 1996). If these were the first human-induced phase shifts in the system, they occurred thousands of years after first human contact and appear to have been localized and shortduration events (Table 4). After European contact, traditional fishing economies of Native American peoples were severely disrupted and effectively ended as their populations were decimated by old-world disease epidemics and colonial oppression (Tegner \& Dayton 2000; Erlandson \& Rick 2002).

The maritime fur trade functionally eliminated sea otters from southern California by the early 1800s (Tegner \& Dayton 2000). However, widespread phase shifts to the deforested state were not observed until 150 years later (Tegner et al. 1996a; Fig. 6). This apparent lag timing between local sea otter extinction and urchin-induced phase shifts in kelp forest probably resulted from the buffering influences of alternate predators, herbivores and competitors (Table 1; Cowen 1983; Tegner \& Levin 1983; Schmitt 1987). Spiny lobsters and sheephead are both subtropical generalist predators that feed on sea urchins and reach their northern range limits in southern California (Dayton et al. 1998; Tegner \& Dayton 2000). Sheephead became larger and more abundant after Native Americans stopped fishing on them. It is also possible that their populations along with that of spiny lobsters increased in abundance following the sea otter's 
demise. Several species of abalone (Haliotis spp.; Table 1) share food and habitat with sea urchins and these competitors may also have kept urchin populations in check (Tegner \& Levin 1983). Released from predation by otters and Native Americans, populations of several abalone species expanded and became targets of a new fishery beginning in 1850 (Cox 1962; Tegner \& Dayton 2000).

During the mid-20th century, intensified fishing pressure on the remaining predators (spiny lobster and sheephead) and herbivorous competitors (five species of abalones) may have relaxed the predation and competition that had been controlling sea urchin populations and phase shifts became widespread for a relatively brief period of time (Fig. 4). Spiny lobster landings peaked in 1894 , and then stabilized at lower levels. However, the larger lobsters, those most adept at killing adult sea urchins (Tegner \& Levin 1983), had become rare. Fishing pressure on large male sheephead increased after the advent of skin diving in the 1940s and accelerated in the 1950s with the loss of alternative targets (Dayton et al. 1998). Abalone harvests accelerated in the 1950 s, causing widespread population declines by the late 1960s such that these grazers are also now functionally extinct. El Niño events, pollution discharge and sedimentation accelerated the loss of kelp, which, along with an increase in destructive urchin grazing, resulted in a phase shift to a largely kelp-free state in the 1950s and 1960s (Fig. 6). Finally, a sea urchin fishery developed and expanded rapidly in the early 1970s, reducing grazing pressure in some areas (Tegner \& Dayton 1991). Commercial harvesting reduced the distribution, abundance, and body size of exploited urchin stocks, leading toward another phase shift back to a forested state. In 1988, a market developed for live sheephead, which resulted in their virtual elimination as a predator in this system (Tegner \& Dayton 2000). Thus the diversity of functionally important species in southern California continues to decline, and, in time this could make the system as a whole less resistant to phase shifts. The few breaks in the canopy kelp that occurred since 1965 resulted from strong storms related to El Niño or La Niña events (Fig. 6) or occasional outbreaks of small herbivorous crustaceans released from their predators due to $\mathrm{El}$ Niño southern oscillation (ENSO) changes in coastal oceanography. Intense storms in 1983 and 1988 reduced the density of the three dominant kelp genera (Macrocystis, Pterygophora and Laminaria; Fig. 7), but recovery was rapid due to high recruitment into the breaks in the Macrocystis canopy (Tegner et al. 1997). In general, the greater diversity of southern California kelp forests, including urchin predators no longer harvested by Native Americans after Spanish colonization, may have buffered the phase shift to a deforested state and facilitated recovery from physical (oceanographic) disturbances. However, this system has experienced serial trophic-level dysfunction, beginning with sea otters and more recently including virtually all other functionallyimportant predators and herbivores.

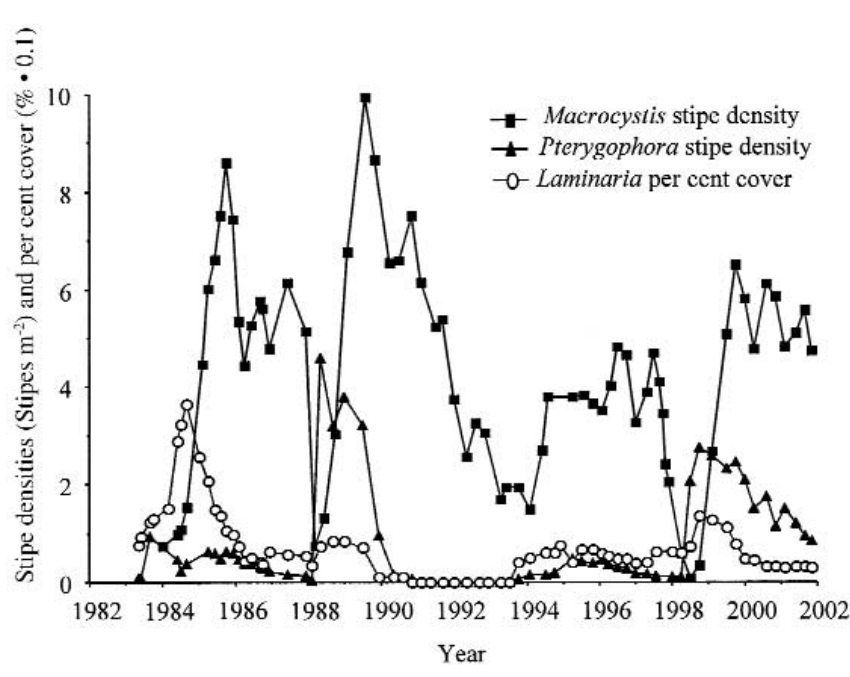

Figure 7 Temporal trends in the kelp forest of Point Loma, California, USA, 1983-1996 at $12 \mathrm{~m}$ depth (from Tegner et al. 1996a). Population density data are shown for canopy (Macrocystis) and stipitate (Pterygophora) kelps. Percentage cover data are shown for the prostrate kelp Laminaria.

\section{Biodiversity, trophic cascades and rates and consequences of kelp deforestation}

In the North American case studies, the extirpation of predators led to increased herbivory by sea urchins resulting in kelp deforestation at local to widespread spatial scales (Fig. 2). In the western North Atlantic and Alaska, where predator diversity is low (Table 1), the transition between kelp forests and coralline communities was rapid, frequent (Fig. 5), widespread and in some cases long-lasting (Table 4). These patterns differ from southern California, where the diversities of predators, herbivores and kelps are high (Table 1), deforestation events have been rare or patchy in space and short in duration (Harrold \& Reed 1985), and no single dominant sea urchin predator exists (Fig. 6; Tegner \& Dayton 2000). The biodiversity within functional nodes, such as trophic levels, is critical to the structure and functioning of kelp forest ecosystems. Nevertheless, even the most diverse systems can and are losing their functional diversity as overfishing reduces the ecologically effective population densities of important species rendering them ecologically-extinct (Estes et al. 1989). All of this suggests that the fragility and rate of change in kelp forest ecosystems may depend on local biodiversity. It remains an open question whether diverse kelp forests will persist or if serial disassembly and instability will inevitably result.

The consequences of kelp deforestation can affect surrounding marine and terrestrial habitats. Drift from giant kelp (Macrocystis pyrifera) dominates nearshore-produced phytodetritus in the Southern California bight, contributing between 60 and $99 \%$ of beach-cast autotrophic detritus (Zobell 1971). Similar estimates were made for areas adjacent 
to kelp forests of eastern Nova Scotia (Mann 2000). Offshore contributions are facilitated by gas-filled floats and stipes which, when adult sporophytes are detached from the bottom due to grazing or physical disturbance, provide for longdistance dispersal by rafting (Harrold \& Lisin 1989; Hobday 2000). When floating kelp rafts are deposited on the shore, the floats break, and they wash into shallow nearshore habitats and ultimately into offshore basins (Graham et al. 2003). Secondary productivity of both shallow (Vetter 1995) and deep-sea (Harrold et al. 1998) soft-sediment systems is consequently driven in a large part by allochthonous food subsidies from regional kelp resources. Kelp detritus can also make its way into nearby intertidal food webs through either the capture of fine kelp particles by filter feeders (for example, mussels [Duggins et al. 1989] or clams [Soares et al. 1997]) or large pieces of drift kelp by limpets (Bustamante $e t$ al. 1995; Bustamante \& Branch 1996) and sea urchins (Day \& Branch 2002). Kelp detritus enhances the inherently low productivity of terrestrial ecosystems on arid islands (Polis \& Hurd 1996). During dry years, carbon and nitrogen from marine bird and mammal faeces and beach cast marine detritus fuel terrestrial productivity, with the greatest impact on islands with large ratios of shoreline to area (Graham et al. 2003). The importance of marine subsidies lessens during rainy years when high precipitation increases terrestrial production. Excellent examples are found in southern California where numerous islands of low productivity (for example, the Channel Islands) are embedded within a highly productive marine system. In addition to localized areas of high accumulation of guano and pinniped excrement, the shoreline is loaded with large quantities of kelp detritus (Graham et al. 2003).

\section{POTENTIAL STATES IN 2025}

\section{Extrapolation of known trends}

It is likely that climate change, human population growth, coastal development, oil spills, fisheries-induced impacts, and invasions of non-native species, will continue and possibly accelerate over the 2025 time horizon. All of these may well contribute to the continuing disassembly of kelp forest ecosystems. It is difficult to extrapolate known trends into the future, because non-linear thresholds and complex interactions can cause ecosystems to behave unpredictably (see Scheffer $e t$ al. 2001). Some activities may change the nature of functional relationships. For example, overfishing of predators of sea urchins can cause kelp forests to decline, but overfishing of sea urchins themselves can have the reverse effect (discussed above). Since the primary market for sea urchin roe is Japan, the currency value of the Yen could regulate fishing pressure on this driver of many kelp forest ecosystems. However it is impossible to predict how global economic markets will evolve over the next several decades.

While global temperature is expected to increase over the period to 2025, related patterns of droughts, fires, heat waves, storms and precipitation are expected to increase in some areas but decline in others (Houghton et al. 1996). This underscores the limitations of attempting to generalize about kelp forest ecosystems globally. However, by considering the future of the three case studies described above, we may gain insight into some of the potential risks that befall these ecosystems with widely varying diversities and scales in space and time. Our review considers trends from the largest spatial and temporal scales to the smallest.

Ocean-climate change: global warming, regime shifts and ENSO

Ocean temperature regulates the physiology and biogeography of marine algae (Adey \& Steneck 2001). Global warming, regime shifts and ENSOs are climate-driven thermal effects that can impact kelp forest ecosystems at a wide range of intensities that operate at several temporal and spatial scales. Global warming operates at the largest temporal and spatial scales (see Fig. 8), but the projected changes over the next two decades are modest compared to ocean-basin-scale regime shifts. Regime shifts cause temperature fluctuations nearly an order of magnitude greater and persist at the temporal scale of decades (Fig. 9). Superimposed on both of those changes are ENSOs, which can cause the greatest temperature anomalies but impact coastal zones at smaller scales and over periods of only a year or two. The interaction among these three ocean climate effects is complicated because each varies differently in space and time. However, when two or more thermal anomalies coincide, the compounded perturbations to kelp forests can be staggering (Paine et al. 1998).

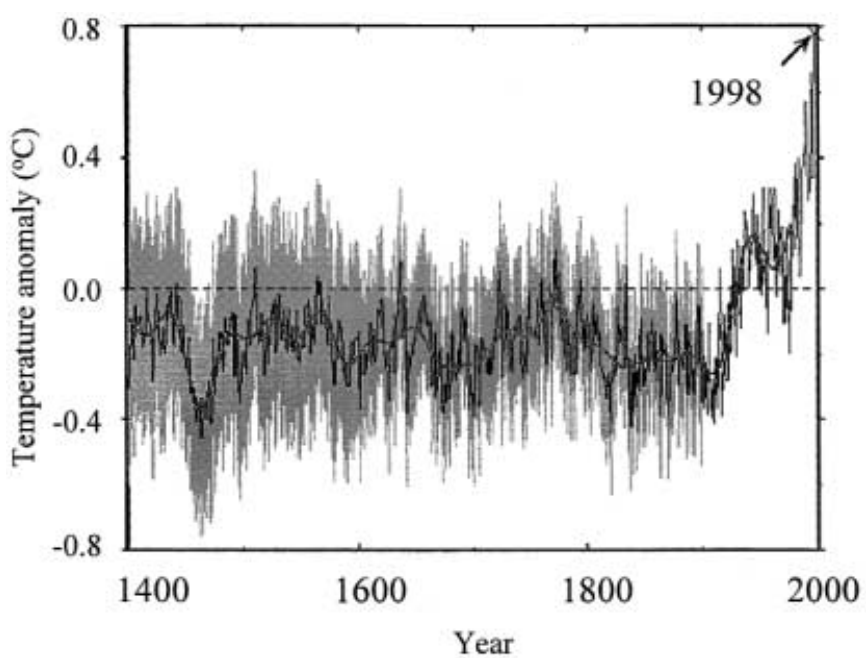

Figure 8 Temperature trends over the past 600 years in the Northern Hemisphere based on multiproxies of palaeoclimates from atmospheric and oceanographic sources (Mann et al. 1998). The average temperature over the 600 -year period is $0.0^{\circ} \mathrm{C}$. Longterm trends are evident in the average trends in 50 year running means. 


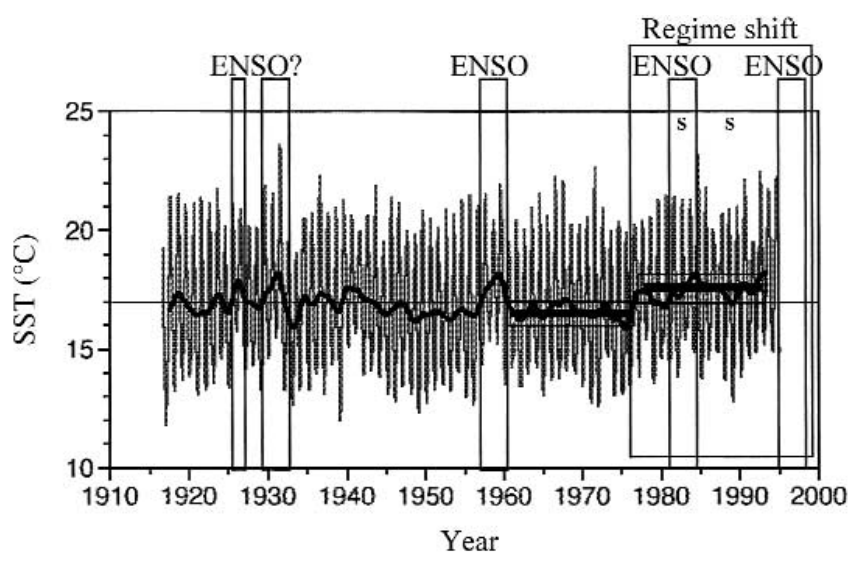

Figure 9 Recent decadal to annual thermal sea surface temperatures (SST) for southern California (from McGowan et al. 1998). The variable bold line over the duration of the time interval indicates the 27-month running mean. The dark horizontal lines indicate regime-shift averages of mean temperature. Superimposed boxes identify periods of regime shift and ENSOs. S = intense storms (Seymour et al. 1989; Dayton et al. 1999).

Mean annual temperature in the Northern Hemisphere has increased by $0.8^{\circ} \mathrm{C}$ over the past century (Mann et al. 1998 ) and is expected to increase by $0.28^{\circ}-0.58^{\circ} \mathrm{C}$ globally by 2025 (Houghton et al. 1996). The rate and magnitude of global temperature rise during the past century is unprecedented over at least the past 600 years (Fig. 8; Mann et al. 1998). As a group, kelps are limited to coldwater coastal zones (Fig. 1). Thus kelps living close to their upper thermal limits will be likely to recede to higher latitudes during protracted warming periods.

Temperature effects are complicated by a variety of ocean-atmosphere interactions. While the long-term frequency of strong storms in the eastern North Pacific Ocean has changed relatively little since AD 1625 (Enfield 1988), recently the frequency and intensity of extreme cyclones have increased markedly (Graham \& Diaz 2001). Two storms in the 1980s were described as storms of the century (Seymour et al. 1989; Fig. 9). The recent steady (rather than stepwise) increase in storm activity may be related to rising sea surface temperatures in the western tropical Pacific (Graham \& Diaz 2001). Thus, abiotic storm-induced disturbances of kelp forests could increase commensurate with global warming (Fig. 9). However, kelp forests usually recover rapidly from such disturbances (Tegner et al. 1997).

In $1982-1983$, a $4-5^{\circ} \mathrm{C}$ ENSO warming halted coastal upwelling and created one of the most severe disturbances of a giant kelp forest ever documented (Paine et al. 1998). Nitrogen concentrations in seawater influence the welfare of kelps and vary with ocean temperature (Gerard 1997). When surface waters stratify due to ENSO or as a result of global warming, nitrogen concentrations decline and kelps become nitrogen limited and may die (Dayton et al. 1999). Macrocystis beds are particularly vulnerable because they possess limited nitrogen storage capacity (Gerard 1982). Although nitrogen in groundwater run-off and atmospheric inputs has increased in coastal oceans (Jickells 1998), its impact is modest or undetectable relative to natural fluxes in all but enclosed basins with limited flushing (Jickells 1998). Thus, trends toward increasing temperature and decreasing nitrogen availability in kelp forests are likely to continue.

The combination of warming coastal oceans and increased stratification will be likely to shrink the geographic range of kelp beds living closest to the tropics. Indirect effects of temperature on species that influence kelp forests will be discussed below. Sea level might rise by $20 \mathrm{~cm}$ by the year 2050 as a result of global warming (Houghton et al. 1996). This could cause increased coastal erosion and turbidity, which could then affect kelp depth distribution (Vadas \& Steneck 1988) and possibly other processes such as photosynthesis and recruitment (Graham 1996). Increased sedimentation could also reduce the area of substrate available for kelp settlement.

Regime shifts and ENSO events can cause thermal anomalies of shorter duration but potentially greater impact. In general, ENSO events create warmer sea temperatures along the southern California coast, but the events themselves are somewhat more frequent during regime shifts that themselves create warmer conditions on that coast (Fig. 9). Following El Niño warming comes La Niña cooling (McGowan et al. 1998), which is often associated with violent storms (Tegner \& Dayton 1987, 1991; Seymore et al. 1989; Tegner et al. 1997).

One of the best-studied climatic regime shifts began in the mid-1970s and ended at the end of the 1990s (Steele 1998). If the new regime's duration is similar to the last two (Fig. 9), then cooler than average temperatures and perhaps lower than average ENSO and storm events may be expected over much of the next quarter century (McGowan et al. 1998; Fei Chai, personal communication 2002). In our case studies, only the California system was significantly affected by storms. However the duration of storm impacts is usually brief and system recovery is rapid; thus, they should have little lasting impact (Figs. 6-7; Tegner et al. 1997).

\section{Changing coastal biodiversity: new apex predators and competitors}

Changes in kelp forest biodiversity that affect functional components of kelp beds can disrupt the system in both predictable and unpredictable ways. The most conspicuous changes to the kelp forest result from overfishing key drivers such as apex predators and sea urchins (Estes \& Duggins 1995; Jackson et al. 2001). Reductions in either driver can lead to trophic-level dysfunction, and alternate stable states or large-scale instabilities. For example, extirpation of sea urchin predators led to hyperabundances of sea urchins that have been stable for decades in Alaska (Fig. 3) and remarkably unstable in Nova Scotia (Fig. 5) due to epizootic disease cycles (Scheibling et al. 1999). Arguably, predator loss led to 
hyperabundances of species setting the stage for diseaserelated mass mortalities (McNeill 1976).

Apex predators in pristine kelp forests were probably relatively large vertebrates. Our case studies show a consistent trend of fishing down food webs, such that large vertebrates were often targeted and extirpated relatively rapidly. Today, fish are the most commonly identified predator, but sea otters and sea ducks are also important vertebrate predators in some northern regions (Table 3 ). In all cases these vertebrate predators are smaller in body size and/or fewer in number than they were in the past (i.e. at first human contact). Crustaceans such as spiny lobsters and crabs are among the most important invertebrate predators (Table 3). If we extrapolate from the known trend in the Gulf of Maine case study in which extirpation of large predatory finfish led to the dominance of crabs as apex predators, then it is possible that crab predation elsewhere is the result of a disrupted trophic cascade. Crabs are dominant predators of sea urchin in Japan and in the UK (Table 3). Both of these regions have a long history of fishing that targeted and extirpated coastal groundfish. In Europe, coastal groundfish stocks were fought over in the 13th and 14th centuries and their depletion is thought to have contributed to the development of distant fisheries in Iceland and eventually North America (Kurlansky 1997). Thus it is possible that the very early extirpation of apex predators in Europe's coastal zones led to the rise of crabs as predators just as has more recently happened in the Gulf of Maine (Leland \& Steneck 2001).

Overfishing in kelp forests leads to ecological (Estes et al. 1989) and possibly absolute (Tegner et al. 1996b) extinctions. This loss in biodiversity may make these systems more susceptible to invasion from non-native species (Stachowicz et al. 1999). Recently, kelp forests in the western North Atlantic have been invaded by the green alga Codium fragile, an introduced competitor that could replace the kelp species in this region, which have a long history of resilience and dominance (Fig 5; Chapman \& Johnson 1990; R.E. Scheibling, personal communication 2001). The possible replacement of Laminaria sp. by Codium sp. requires sufficient breaks in the canopy for the latter species to take hold. This has been facilitated by the introduction in the 1970s of a non-native encrusting bryozoan that coats, embrittles and opens the kelp canopy every summer (Lambert et al. 1992; Levin et al. 2003). However, these two invaders join a long list of invading species that have become important players in kelp forest ecosystems of the western North Atlantic (Steneck \& Carlton 2001). Species such as the common periwinkle (Littorina littorea) and the green and shore crabs (Carcinus maenas, Hemigrapsus sanguineus) have not only invaded, but in many cases have come to dominate the ecosystem (Steneck \& Carlton 2001). Whereas other marine systems have a history of invasion, few have seen the largescale changes in dominance evident in the western North Atlantic. The successful series of invasions there stands in stark contrast with patterns observed in the species-rich southern California kelp forests, where introduced species generally remain subordinate to native dominants.

\section{Declining water quality}

Coastal development often reduces the permeability of soil in the watershed resulting in greater run-off and increased turbidity from plankton and particulates. If this occurred, the areal extent of kelp beds would shrink as the areal extent of the photic zone and thus their habitable area declined. Extremely low iron availability may reduce the potential productivity of kelp. If nitrogen compounds increase due to run-off, sewage disposal from population centres, or nitrogen input from atmospheric sources, nutrient availability could increase. However, human inputs of nutrients are evident only in coastal areas of restricted water exchange and most coastal zones 'appear to be still dominated by large inputs from the open ocean and there is little evidence of anthropogenic perturbations' ( Jickells 1998).

In heavily urbanized areas of Japan, terrestrial deforestation and damming of rivers is thought to starve coastal zones of the iron and humic substances necessary for kelp development (Suzuki et al. 1995; Matsunaga et al. 1999). This is hypothesized to create a phase shift from kelp dominance to coralline dominance without any changes in herbivory, sea temperature or macro-nutrients (Matsunaga et al. 1999). We know of no other urbanized area (for example, southern California or Boston, Massachusetts, USA) where crustose coralline algae dominate shallow rocky shores without herbivore populations.

Point-source pollution is often very conspicuous, but rarely has it resulted in a serious deforestation of kelp ecosystems. A broken sewer in southern California in the late 1950s and early 1960s may have resulted in the increase of sea urchins and the decline in Macrocystis canopy there during that period (see Fig. 6 and previous discussion), but the impact was confounded by several other factors. The massive Exxon Valdez oil spill in 1989 occurred in the vicinity of kelp forests of south-west Alaska. However, kelps were minimally impacted and they recovered rapidly (Dean \& Jewett 2001). For most components of the kelp forests, full recovery took two years or less (Dean \& Jewett 2001). Oil covered sea otters in the spill area, and while the accounts of impacts on them are debated (Paine et al. 1996), the greatest impact was a decrease from around 5 otters $\mathrm{km}^{-1}$ of shoreline to between 2-3 otters $\mathrm{km}^{-1}$ (Paine et al. 1996). Otters were never absent from the system, but they may have suffered a long-term and lingering impact from oil in at least parts of spill area in Prince William Sound (Monson et al. 2000). While otters and other airbreathing predators may be most susceptible to oil spills, with effects similar to overfishing, that may not hold for apex predators that are fish. For example, following the Exxon Valdez oil spill, mortality reports estimated thousands of sea otters and sea birds died, but listed fewer than 10 dead fish from the event (Paine et al. 1996). Thus fish may be inherently less susceptible to oil spills, but they are by no means immune. 
Given the likelihood that both non-point source and point-source pollution are likely to increase with increased human population growth, water quality is expected to continue to decline. We do not yet know if thresholds of accelerated mortality exist for declining water quality as they apparently do for increased fishing pressure. However, it is currently top-down impacts on ecosystem drivers (such as sea urchins and their predators) that most consistently denude kelp forests.

\section{CONCLUSIONS AND MANAGEMENT}

Kelps are the largest bottom-dwelling organisms to occupy the euphotic zone. Their size and photosynthesis to biomass ratio constrain their distribution globally and locally. Kelps are among the shallowest of the subtidal macrophytes. Kelp forests fail to develop at high latitudes due to light limitations and at low latitudes due to limitations in nutrients, high sea temperatures and competition from other macrophytes. In shallow mid-latitude rocky marine shores worldwide, phyletically diverse, structurally complex and highly productive kelp forests develop. These are uniquely capable of altering local oceanography and ecology by dampening wave surge, shading the sea floor with their canopy, providing a physical habitat for organisms above the benthic boundary-layer and by distributing trophic resources to surrounding habitats. In this context, the three kelp forest case studies from North America represent ecosystems along a continuum of natural biodiversity and human interactions. In each system we reviewed archaeological literature in an attempt to reconstruct an ecological baseline for the structure and function of kelp forests prior to contact with modern humans.

Consumer animals structure kelp forest interactions via two primary 'drivers', namely (1) herbivory by sea urchins and (2) carnivory from predators of sea urchins. Other forcing functions can be important. For example, kelps are prone to destruction and thinning by storms and competitors. Further, their growth and survival are sensitive to temperature, light and nutrient availability. However, the spatial scale and magnitude of these impacts on kelp forests are small relative to those of the consumers. Kelp deforestation worldwide results from sea urchin grazing, which is controlled by predation in kelp forests where human harvesting impacts have been minimal.

Kelp forests of the eastern Pacific may have facilitated an early coastal migration of humans into the Americas. The concentration and high productivity of vertebrates and invertebrates along this coast would have provided early human settlers with a stable source of food between 15000 and 10000 years ago. Archaeological data indicate that coastal settlements exploited organisms associated with kelp forests for thousands of years and this occasionally resulted in the localized loss of apex predators, outbreaks of sea urchin populations and deforestation. However, these human impacts on kelp bed systems were probably localized and relatively ephemeral.
Over the past two centuries, the commercial exploitation of kelp forest consumers led to the extirpation of sea urchin predators such as the sea otter in the North Pacific and groundfish such as Atlantic cod in the North Atlantic. In those systems, sea urchin abundances increased and kelp forests were denuded over vast stretches of coast. In the southern California system, the high diversities of predators, herbivores and kelp appear to have buffered this system from systemic deforestation.

Biodiversity of kelp forests may also help resist invasion of non-native species. In the species-depauperate western North Atlantic, introduced algal competitors carpet the benthos and threaten the dominance of kelp. Other introduced herbivores and predators have taken hold and have increased to dominate components of the system.

Global and regional climate changes have measurable impacts on kelp forest ecosystems. Increasing frequencies of ENSO events, oceanographic regime shifts and violent storms cause deforestation. This, in combination with the serial loss in biodiversity from overfishing, appears to be the greatest threat to structure and functioning of these systems over the 2025 time horizon.

Management for the conservation of kelp forest ecosystems should focus on restoring biodiversity and especially on minimizing fishing on predators. In particular, species such as sea otters, sheephead and cod should be restored to fulfil their functional role in the Alaska, California and western North Atlantic systems, respectively. While sea otters are already protected, other commercially valuable species such as Atlantic cod will be unlikely to be preserved for this ecosystem role that they perform. Ultimately, human values and political will determine the conservation agenda. Significant investment in education for stakeholders, the general public and policy makers will be necessary for this conservation goal to succeed.

\section{ACKNOWLEDGEMENTS}

This work was conducted as part of the Long-Term Ecological Records of Marine Environments, Populations and Communities Working Group developed by Jeremy B. C. Jackson and supported by the National Center for Ecological Analysis and Synthesis (funded by NSF grant DEB-0072909, the University of California, and the University of California, Santa Barbara). Additional support was provided by The Pew Foundation for Marine Conservation (to RSS, JAE and MJT), NOAA's Sea Grant program to the University of Maine (to RSS), a University of California faculty fellowship (to MHG) and the National Undersea Research Program's National Research Center at the University of Connecticut at Avery Point (Grant No. NA46RU0146 and UCAZP 94-121 to RSS). Colleagues shared insights and unpublished data for several kelp forest ecosystems worldwide. In particular we thank, Neil Andrew (New South Wales, Australia), Russ Babcock (New Zealand), George Branch (South Africa), Bob Cowen (California), Ken 
Dunton (Arctic Alaska), Daisuke Fujita (Japan), Craig Johnson (Tasmania, Australia), Pato Ojeda (Chile), Bob Scheibling (Nova Scotia), Peter Steinberg (New South Wales, Australia) and Julio Vasquez (Chile). Rodrigo Bustamante, Geoff Jones, Paul Dayton, Nicholas Polunin and an anonymous reviewer provided terrific guidance for revising this paper. Amanda Leland, John Vavrinec and Chantale Bégin critiqued the manuscript. Sarah Maki provided technical assistance. To all we are grateful.

\section{References}

Abbott, I.A. \& Hollenberg, G.J. (1976) Marine Algae of California. Stanford, CA, USA: Stanford University Press.

Adey, W.H. (1964) The genus Phymatolithon in the Gulf of Maine. Hydrobiologia 24: 377-420.

Adey, W.H. \& Steneck, R.S. (2001) Thermogeography over time creates biogeographic regions: a temperature/space/time-integrated model and an abundance-weighted test for benthic marine algae. Fournal of Phycology 37: 677-698.

Anderson, R.J., Carrick, P., Levitt, G.J. \& Share, A. (1997) Holdfasts of adult kelp Ecklonia maxima provide refuges from grazing for recruitment of juvenile kelps. Marine Ecology Progress Series 159: 265-273.

Andrew, N.L. (1993) Spatial heterogeneity, sea urchin grazing, and habitat structure on reefs in temperate Australia. Ecology 74: 292-302.

Andrew, N.L. (1994) Survival of kelp adjacent to areas grazed by sea urchins in New South Wales, Australia. Australian fournal of Ecology 19: 466-472.

Andrew, N.L. \& Choat, J.H. (1982) The influence of predation and nonspecific adults on the abundance of juvenile Evechinus chloroticus (Echinodea: Echinodermata). Oecologia 54: 80-87.

Andrew, N.L. \& O'Neill, A.L. (2000) Large-scale patterns in habitat structure on subtidal rocky reefs in New South Wales. Marine and Freshwater Research 51: 255-263.

Andrew, N.L. \& Underwood, A.J. (1993) Density-dependent foraging in the sea urchin Centrostephanus rodgersii on shallow subtidal reefs in New South Wales, Australia. Marine Ecology Progress Series 99: 89-98.

Babcock, R.C, Kelly, S., Shears, N.T., Walker, J.W. \& Willis, T.J. (1999) Changes in community structure in temperate marine reserves. Marine Ecology Progress Series 189: 125-134.

Barrales, H.L. \& Lobban, C. (1975) The comparative ecology of Macrocystis pyrifera with emphasis on the forests of Chubut Argentina. Ecology 63: 657-677.

Bernstein, B.B. \& Jung, N. (1980) Selective pressures and coevolution in a kelp canopy community in Southern California. Ecological Monographs 49: 335-355.

Bernstein, B.B., \& Welsford (1982) An assessment of feasibility of using high-calcium quicklime as an experimental tool for research into kelp bed/sea urchin ecosystems in Nova Scotia. Canadian Technical Report of Fisheries and Aquatic Sciences 968: 1-51.

Bologna, P.A.X. \& Steneck, R.S. (1993) Kelp beds as habitat for American lobster Homarus americanus. Marine Ecology Progress Series 100:127-134.

Bolton, J.J. (1996) Patterns of species diversity and endemism in comparable temperate brown algal floras. Hydrobiologia 326/327: $173-178$.

Bolton, J.J. \& Anderson, R.J. (1987) Temperature tolerances of two southern African Ecklonia species (Alariaceae: Laminariales) and of hybrids between them. Marine Biology 96: 293-297.

Bourque, B.J. (1995) Diversity And Complexity In Prehistoric Maritime Societies: A Gulf Of Maine Perspective. New York, USA: Plenum Press.

Bourque, B.J. (2001) Twelve Thousand Years: American Indians In Maine. Lincoln, Nebraska, USA: University of Nebraska Press.

Breen, P.A. \& Mann, K.H. (1976) Changing lobster abundance and the destruction of kelp beds by sea urchins. Marine Biology 34: 137-142.

Bustamante, R.H. \& Branch, G.M. (1996) The dependence of intertidal consumers on kelp-derived organic matter on the west coast of South Africa. Journal of Experimental Marine Biology and Ecology 196: 1-28.

Bustamante, R.H., Branch, G.M. \& Eekhout, S. (1995) Maintenance of an exceptional intertidal grazer biomass in South Africa: subsidy by subtidal kelps. Ecology 76: 2314-2329.

Bustnes, J.O., Lonne, O.J., Skjoldal, H.R., Hopkins, C., Erikstad, K.E. \& Leinass, H.P. (1995) Sea ducks as predators on sea urchins in a northern kelp forest. In: Ecology of Fjords and Coastal Waters, ed. H.R. Skjoldal, C. Hopkins \& K.E. Erikstad, pp. 599-608. Amsterdam, the Netherlands: Elsevier Science.

Castilla, J.C. \& Moreno, C.A. (1982) Sea urchins and Macrocystis pyrifera: experimental test of their ecological relations in southern Chile. Proceedings of the International Echinoderm Conference, Tampa Bay, Florida, USA, ed. J.M. Lawrence, pp. 257-263. Rotterdam, the Netherlands: Balkema.

Chapman, A.R.O. (1986) Age versus stage: an analysis of age- and size-specific mortality and reproduction in a population of Laminaria longicruris Pyl. Fournal of Experimental Marine Biology and Ecology 97: 113-122.

Chapman, A.R.O. \& Craigie, J.S. (1977) Seasonal growth in Laminaria longicruris: relations with dissolved inorganic nutrients and internal reserves of nitrogen. Marine Biology 40: 197-205.

Chapman, A.R.O. \& Johnson, C.R. (1990) Disturbance and organization of macroalgal assemblages in the Northwestern Atlantic. Hydrobiologia 192: 77-121.

Choat, J.H. \& Ayling, A.M. (1987) The relationship between habitat and fish faunas of New Zealand reefs. Fournal of Experimental Marine Biology and Ecology 110: 228-284.

Choat, J.H. \& Schiel, D.R. (1982) Patterns of distribution and abundance of large brown algae and invertebrate herbivores in subtidal regions of northern New Zealand. Fournal of Experimental Marine Biology and Ecology 60: 129-162.

Clementz, M. (2002) The evolution of herbivorous marine mammals: ecological and physiological transitions during the evolution of the orders Sirenia and Desmostylia. Ph.D. dissertation, Department of Earth Sciences, University of California at Santa Cruz, USA.

Cole, R.G. \& Babcock, R.C. (1996) Mass mortality of a dominant kelp (Laminariales) at Goat Island, North-eastern New Zealand. Marine Freshwater Resource 47: 907-911.

Cole, R.G. \& Syms, C. (1999) Using spatial pattern analysis to distinguish causes of mortality: an example from kelp in northeastern New Zealand. Fournal of Ecology 87: 963-972,

Conkling, P.W. \& Ames, T. (1996) Penobscot fisheries in the 20th century. In: Penobscot: the Forest River and Bay, ed. D.D. Platt, pp. 46-65. Rockland, ME, USA: Island Institute.

Cowen, R.K. (1983) The effect of sheephead (Semicossyphus pulcher) predation on red sea urchin populations: an experimental analysis. Oecologia 58: 249-255.

Cox, K.W. (1962) California abalones, family Haliotidae. California 
Department of Fish and Game, Fishery Bulletin 118, California, USA: $133 \mathrm{pp}$.

Darwin, C. (1909) The Voyage of the Beagle. The Harvard Classics Volume 29. New York, USA: P.F. Collier \& Son Company.

Day, E. \& Branch, G.M. (2002) Effects of sea urchins (Parechinus angulosus) on recruits and juveniles of abalone (Haliotis midae). Ecological Monographs 72: 133-149.

Dayton, P.K. (1975) Experimental studies of algal-canopy interactions in a sea otter dominated kelp community at Amchitka Island, Alaska. Fishery Bulletin, United States 73: 230-237.

Dayton, P.K. (1985a) Ecology of kelp communities. Annual Reviem Ecology Systems 16: 215-245.

Dayton, P.K. (1985b) The structure and regulation of some South American kelp communities. Ecological Monographs 55: 447-468.

Dayton, P.K. \& Tegner, M.J. (1990) Bottoms below troubled waters: benthic impacts of the 1982-84 El Niño in the temperate zone. In: Ecological Consequences of the 1982-83 El Niño to Marine Life, ed. P.W. Glynn, Elsevier Oceanography Series No. 52, pp. 433-472. Amsterdam, the Netherlands: Elsevier: 563 pp.

Dayton, P.K., Currie, V., Gerrodete, T., Keller, B.D., Rosenthal, R., \& Ven Tresca, D. (1984) Patch dynamics and stability of some California kelp communities. Ecological Monographs 54: 253-289.

Dayton, P.K., Tegner, M.J. Edwards, P.B. \& Riser, K.L. (1998) Sliding baselines, ghosts, and reduced expectations in kelp forest communities. Ecological Applications 8: 309-322.

Dayton, P.K., Tegner, M.J., Edwards, P.B. \& Riser, K.L. (1999) Temporal and spatial scales of kelp demography: the role of oceanography climate. Ecological Monographs 69: 219-250.

Dean, T.A. \& Jewett, S.C. (2001) Habitat-specific recovery of shallow subtidal communities following the Exxon Valdez oil spill. Ecological Applications 11: 1456-1471.

Domning, D.P. (1972) Steller's sea cow and the origin of North Pacific aboriginal whaling. Syesis 5: 187-189.

Duggins, D.O. (1980) Kelp beds and sea otters: an experimental approach. Ecology 61: 447-453.

Duggins, D.O. (1983) Starfish predation and the creation of mosaic patterns in a kelp dominated community. Ecology 64: 1610 1619.

Duggins, D.O., Eckman, J.E. \& Sewell, A.T. (1990) Ecology of understory kelp environments. II. Effects of kelps on recruitment of benthic invertebrates. Fournal of Experimental Marine Biology and Ecology 143: 27-45.

Duggins, D.O., Simenstad, C.A. \& Estes, J.A. (1989) Magnification of secondary production by kelp detritus in coastal marine ecosystems. Science 245: 101-232.

Dunton, K.H. (1990) Growth and production in Laminaria solidungula: relation to continuous underwater light levels in the Alaskan High Arctic. Marine Biology 106: 297-304.

Dunton, K.H. \& Dayton, P.K. (1995) The biology of high latitude kelp. In: Ecology of Fjords and Coastal Waters, ed. H.R. Skjoldal, C. Hopkins, K.E. Erikstad \& H.P. Leinass, pp. 499-507. Amsterdam, the Netherlands: Elsevier Science.

Dunton, K.H. \& Schell, D.M. (1987) Dependence of consumers on macroalgal (Laminaria solidungula) carbon in an Arctic kelp community: delta-carbon-13 evidence. Marine Biology 93: 615-626.

Durham, J.W. \& MacNeil, F.S. (1967) Cenozoic migrations of marine invertebrates through the Bering Strait region. In: The Bering Land Bridge, ed. D.M. Hopkins, pp. 326-349. Stanford, CA, USA: Stanford University Press.

Ebeling, A.W., Laur, D.R. \& Rowley, R.J. (1985) Severe storm disturbance and reversal of community structure in a southern California kelp forest. Marine Biology 84: 287-294.

Ebling, F.S., Hawkins, A.D., Kitching, J.A., Muntz, P. \& Pratt, V.M. (1966) The ecology of Lough Ine XVI. Predation and diurnal migrations in Paracentrotus community. Fournal of Animal Ecology 35: 559-566.

Edelstein, T., Craigie, J.S. \& McLachlan, J. (1969) Preliminary survey of the sublittoral flora of Halifax county. Fournal of Fisheries Research Board of Canada 26: 2703-2713.

Enfield, D.B. (1988) Is El Niño becoming more common? Oceanography 59: 123-127.

Erlandson, J.M. (2001) Anatomically modern humans, maritime voyaging, and the Pleistocene colonization of the Americas. In: The First Americans: The Pleistocene Colonization of the Nem World, ed. N.G. Jablonski, pp. 1-9. San Francisco, CA, USA: California Academy of Sciences.

Erlandson, J.M. \& Rick, T.C. (2002) Human impacts on ancient environments: a case study from California's Northern Channel Islands. In: The Archaeology of Insularity: Examining the Past in Island Environments, ed. S.M. Fitzpatrick (in press). New York, USA: Praeger.

Erlandson, J.M., Kennett, D.J., Ingram, B.L., Guthrie, D.A., Morris, D.P., Tveskov, M.A., West, G.J. \& Walker, P.L. (1996) An archaeological and palaeontological chronology for Daisy Cove (CA-SMI-261), San Miguel Island, California. Radiocarbon 38: $355-373$.

Estes, J.A. \& Duggins, D.O. (1995) Sea otters and kelp forests in Alaska: generality and variation in a community ecological paradigm. Ecological Monographs 65: 75-100.

Estes, J.A. \& Steinberg, P.D. (1988) Predation, herbivory, and kelp evolution. Paleobiology 14: 19-36.

Estes, J.A., Duggins, D.O. \& Rathbun, G.B. (1989) The ecology of extinctions in kelp forest communities. Conservation Biology 3: $252-264$.

Estes, J.A., Tinker, M.T., Williams, T.M. \& Doak, D.F. (1998) Killer whale predation on sea otters linking oceanic and nearshore ecosystems. Science 282: 473-476.

Foster, M.S. (1990) Organization of macroalgal assemblages in the Northeast Pacific: the assumption of homogeneity and the illusion of generality. Hydrobiologia 192: 21-33.

Foster, M. \& Schiel, D.R. (1985) The ecology of giant kelp forests in California: a community profile. US Fish and Wildlife Service Biological Report 85 (7.2), USA: 152 pp.

Fujita, D. (1987) The report of interviews with fishermen on Isoyake in Taisei-cho, Hokkaido. Suisanzoshoku 35: 135138.

Fujita, D. (1998) Strongylocentrotid sea urchin-dominated barren grounds on the Sea of Japan coast of northern Japan. In: Echinoderms, ed. R. Mooi \& M. Telford, pp. 659-664. Rotterdam, the Netherlands: A.A. Balkema.

Gagne, J.A., Mann, K.H. \& Chapman, A.R.O. (1982) Seasonal patterns of growth and storage in Laminaria longicruris in relation to differing patterns of availability of nitrogen in the water. Marine Biology 69: 91-101.

Gaines, S.D. \& Roughgarden, J. (1987) Fish in offshore kelp forests affect recruitment to intertidal barnacle populations. Science $\mathbf{2 3 5}$ : 479-481.

Gerard, V.A. (1982) Growth and utilization of internal nitrogen reserves by the giant kelp Macrocystis pyrifera in a low nitrogen environment. Marine Biology 66: 27-35.

Gerard, V.A. (1997) The role of nitrogen nutrition in high-tempera- 
ture tolerance of kelp, Laminaria saccharina. Fournal of Phycology 33: $800-810$.

Graham, M.H. (1996) Effect of high irradiance on recruitment of the giant kelp Macrocystis (Phaeophyta) in shallow water. Fournal of Phycology 32: 903-906.

Graham, M.H., Dayton, P.K. \& Erlandson, J.M. (2003) Ice ages and the shifting ecology of temperate coasts. Trends in Ecology and Evolution (in press).

Graham, M.H., Harrold, C., Lisin, S., Light, K. Watanabe, J.M. \& Foster, M.S. (1997) Population dynamics of giant kelp Macrocystis pyrifera along a wave exposure gradient. Marine Ecology Progress Series 148: 269-279.

Graham, N.E. \& Diaz, H.F. (2001) Evidence for intensification of North Pacific winter cyclones since 1948. Bulletin of American Meteorological Society 82: 1869-1893.

Hagen, N.T. (1983) Destructive grazing of kelp beds by sea urchins in Vestfjorden, northern Norway. Sarsia 68: 177-190.

Harris, L.G., Ebeling, A.W., Laur, D.R. \& Rawley, R.J. (1984) Community recovery following storm damage: a case of facilitation in primary succession. Science 224: 1336-1338.

Harrold, C. \& Lisin, S. (1989) Radio-tracking rafts of giant kelp: local production and regional transport. Fournal of Experimental Marine Biology and Ecology 30: 237-252.

Harrold, C. \& Reed, D.C. (1985) Food availability, sea urchin grazing, and kelp forest community structure. Ecology 66: 1160-1169.

Harrold, C., Light, K. \& Lisin, S. (1998) Organic enrichment of submarine-canyon and continental-shelf benthic communities by macroalgal drift imported from nearshore kelp forests. Limnology and Oceanography 43: 669-678.

Hart, M.W. \& Scheibling, R.E. (1988) Heat waves, baby booms, and the destruction of kelp beds by sea urchins. Marine Biology 99: $167-176$.

Hatcher, B.G., Kirkman, H. \& Wood, W. F. (1987) Growth of the kelp Ecklonia radiata near the northern limit of its range in Western Australia. Marine Biology 95: 63-73.

Henley, W.J. \& Dunton, K.H. (1997) Effects of nitrogen supply and continuous darkness on growth and photosynthesis of the arctic kelp Laminaria solidungula. Limnology and Oceanography 42: 209-216.

Hervey, A.B. (1881) Sea Mosses: a Collector's Guide and an Introduction to the Study of the Marine Algae. Salem, MA, USA: S.E. Cassino.

Himmelman, J.H. (1980) The role of the green sea urchin Strongylocentrotus droebachiensis in the rocky subtidal region of Newfoundland. In: Proceedings of The Workshop On The Relationship Between Sea Urchin Grazing And Commercial Plant/Animal Harvesting, ed. J.D. Pringle, G.J. Sharp \& J.F. Caddy, pp. 92-119. Halifax, NS, Canada: Fisheries and Oceans Canada, Resource Branch.

Hjorleifsson, E., Kassa, O. \& Gunnarsson, K., (1995) Grazing of kelp by green sea urchins in Eyyjafjordu, North Iceland. In: Ecology of Fjords and Coastal Waters, ed. H.R. Skjoldal, C. Hopkins, K.K. Erikstad \& H.P. Leinass, pp. 593-597. Amsterdam, the Netherlands: Elsevier Science.

Hobday, A.J. (2000) Abundance and dispersal of drifting kelp Macrocystis pyrifera rafts in the Southern California Bight. Marine Ecology Progress Series 195: 101-116.

Hooper, R. (1980) Observations on algal-grazer interactions in Newfoundland and Labrador. In: Proceedings Of The Workshop On The Relationship Between Sea Urchin Grazing And Commercial
Plant/Animal Harvesting, ed. J.D. Pringle, G.J. Sharp \& J.F. Caddy, pp. 120-124. Halifax, NS, Canada: Fisheries and Oceans Canada, Resource Branch.

Houghton, J.T., Meira Filho, L.G., Bruce, J., Hoesung Lee Callander, B.A., Haites, E. \& Maskell, K. (1996) Climate Change 1995. World Meteorological Organization/Intergovernmental Panel on Climate Change, Cambridge, UK: Cambridge University Press.

Jackson, J.B., Kirby, M.X., Berger, W.H., Bjorndal, K.A., Botsford, L.W., Bourque, B.J., Bradbury, R., Cooke, R., Erlandson, J., Estes, J.A., Hughes, T.P., Kidwell, S., Lange, C.B., Lenihan, H.S., Pandolfi, J.M., Peterson, C.H., Steneck, R.S., Tegner, M.J. \& Warner, R. (2001) Historical overfishing and the recent collapse of coastal ecosystems. Science 293: 629-638.

Jickells, T.D. (1998) Nutrient biogeochemistry of the coastal zone. Science 281: 217-222.

Johnson, C.R. \& Mann, K.H. (1986) The importance of plant defense abilities to the structure of subtidal seaweed communities: the kelp Laminaria longicruris de la Pylaie survives grazing by the snail Lacuna vincta (Montagu) at high populations densities. Fournal of Experimental Marine Biology and Ecology 97: 231-267.

Johnson, C.R. \& Mann, K.H. (1988) Diversity, patterns of adaptation, and stability of Nova Scotian kelp beds. Ecological Monographs 58: 129-154.

Johnson, D.S. \& Skutch, A.F. (1928) Littoral vegetation on a headland of Mt. Desert Island, Maine. I. Submersible or strictly littoral vegetation. Ecology 9: 188-215.

Jones, N.S. \& Kain, J.M. (1967) Subtidal algal colonization following the removal of Echinus. Helgolander Wissenshaftichen Meeresuntersuchungen 15: 160-166.

Jones, R.E. (1967) A Hydrodamalis skull fragment from Monterey Bay, California. Journal of Mammalogy 48: 143-144.

Kain, J.M. (1975) Algal recolonization of some cleared subtidal areas. Fournal of Ecology 63: 739-765.

Kain, J.M. (1979) A view of the genus Laminaria. Oceanographic Marine Biology Annual Reviem 17: 101-161.

Keats, D.W., Steele, D.H. \& South, G.R. (1986) Atlantic wolffish (Anarhichas lupus L.; Pisces: Anarhichidae) predation on green sea urchins (Strongylocentrotus droebachiensis (O. F. Mull.): Echinodermata: Echinoidea) in eastern Newfoundland. Canadian Fournal of Zoology 64: 1920-1925.

Kitching, J.A. \& Ebling, F.J. (1961) The ecology of Lough Ine XI. The control of algae by Paracentrotus lividus (Echinoidea). Fournal of Animal Ecology 30: 373-383.

Kitching, J.A. \& Thain, V.M. (1983) The ecological impact of the sea urchin Paracentrotus lividus (Lamarck) in Lough Ine, Ireland. Philosophical Transactions of the Royal Society of London B 300: 513-552.

Korb, R.E. \& Gerard, V.A. (2000) Effects of concurrent low temperature and low nitrogen supply on polar and temperate seaweeds. Marine Ecology Progress Series 198: 73-82.

Kurlansky, M. (1997) Cod: a Biography of the Fish that Changed the World. New York, USA: Walker and Co.

Lamb, M. \& Zimmerman, M.H. (1964) Marine vegetation of Cape Ann, Essex County, Massachusetts. Rhodora 66: 217-254.

Lambert, W.J., Levin, P.S. \& Berman, J. (1992) Changes in the structure of a New England (USA) kelp bed: the effects of an introduced species? Marine Ecology Progress Series 88: 303-307.

Lawrence, J.M. (1975) On the relationships between marine plants and sea urchins. Oceanography and Marine Biology Annual Reviem 13: 213-286. 
Leighton, D.L., Jones, L.G. \& North, W. (1966) Ecological relationships between giant kelp and sea urchins in southern California. In: Proceedings 5th International Seaweed Symposium Oxford, England, ed. E.G. Young \& J.L. McLachlan, pp. 141-153. Oxford, UK: Pergamon Press.

Leland, A. (2002) A new apex predator in the Gulf of Maine: large, mobile Jonah crabs (Cancer borealis) control benthic community structure. Masters Thesis, University of Maine. Orono Maine, USA.

Leland, A. \& R. S. Steneck. (2001) Are crabs in Maine a new apex predator? Abstracts of the 82nd Annual Meeting of the Western Society of Naturalists, Monterey, CA, USA.

Levin, P. (1991) Effects of microhabitat on recruitment variation in a Gulf of Maine reef fish. Marine Ecology Progress Series 75: 183-189.

Levin, P.S. (1994) Fine-scale temporal variation in recruitment of a temperate demersal fish: the importance of settlement versus post-settlement loss. Oecologia 97: 124-133.

Levin, P.S., Coyer, J.A., Petrik, R. \& Good, T.P. (2003) Community-wide effects of non-indigenous species on temperate rocky reefs. Ecology (in press).

Linley, E.A.S., Newell, R.C. \& Bosma, S.A. (1981) Hetertrophic utilization of mucilage released during fragmentation of kelp (Ecklonia maxima and Laminaria pallida) development of microbial communities associated with the degradation of kelp mucilage. Marine Ecology Progress Series 4: 31-41.

MacFarlane, C. (1952) A survey of certain seaweeds of commercial importance in southwest Nova Scotia. Canadian Fournal of Botany 30: 78-97.

Mann, K.H. (1973) Seaweeds: their productivity and strategy for growth. Science 182: 975-981.

Mann, K.H. (1977) Destruction of kelp beds by sea urchins: a cyclical phenomenon or irreversible degradation? Helgolander Wissenshaftlichen Meeresuntersuchungen 30: 455-467.

Mann, K.H. (2000) Ecology of Coastal Waters, with Implications for Management. Volume 2. Oxford, UK: Blackwell Science: $406 \mathrm{pp}$.

Mann, M.E., Bradley, R.S. \& Hughes, M.K. (1998) Global-scale temperature patterns and climate forcing over the past six centuries. Nature 392: 779-787.

Matsunaga, K., Kawaguchi, T., Suzuki Y. \& Nigi, G. (1999) The role of terrestrial humic substances on the shift of kelp community to crustose coralline algae community of the southern Hokkaido Island in the Japan Sea. Fournal of Experimental Marine Biology and Ecology 241: 193-205.

McGowan, J.A., Cayan, D.R., Dorman, L.M. (1998) Climate-ocean variability and ecosystem response in the Northeast Pacific. Science 281: 210-217.

McNaught, D.C. (1999) The indirect effects of macroalgae and micropredation on the post-settlement success of the green sea urchin in Maine. Ph. D. Dissertation, Department of Oceanography, University of Maine, USA.

McNeill, W.H. (1976) Plagues and Peoples. New York, USA: Anchor Books: 340 pp.

Moe, R. \& Silva, P. (1981) Morphology and taxonomy of Himantothallus (including Phaeoglossum and Phyllogigas), an Antarctic member of the Desmarestiales (Phaeophyceae). Fournal of Phycology 17: 15-29.

Monson, D.H., Doak, D.F., Ballachey, B.E., Johnson, A.M. \& Bodkin, J.L. (2000) Long-term impacts of the Exxon Valdez oil spill on sea otters, assessing through age-dependent mortality patterns. Proceedings of the National Academy of Sciences 97: 6562-6567.

Neushul, M. (1972) Functional interpretation of benthic marine algal morphology. Contributions to the systematics of benthic marine algae of the North Pacific, ed. I.A. Abbott \& M. Kurogi, pp 47-74. Kobe, Japan: Japanese Society of Phycology

Novaczek, I. \& McLachlan, J. (1986) Recolonization by algae of the sublittoral habitat of Halifax County, Nova Scotia, following the demise of sea urchins. Botanica Marina 24: 69-73.

Ohmi, H. (1951) Studies on Isoyake or decrease of seaweeds along the coast of Northern Japan. Bulletin Faculty Fisheries Hakodate, Hokkaido University 2: 109-117.

Ojeda, F. \& Santelices, B. (1984) Ecological dominance of Lessonia nigrescens (Phaeophyta) in central Chile. Marine Ecology Progress Series 19: 83-91.

Paine, R. T. (1980) Food webs: linkages, interaction strength and community infrastructure. Fournal of Animal Ecology 49: 667-685.

Paine, R.T., Ruesink, J.L., Sun, A., Soulanille, E.L., Wonham, M.J., Harley, C.D.G., Brumbaugh, D.R. \& Secord, D.L. (1996) Trouble on oiled waters: lessons from the Exxon Valdez oil spill. Annual Reviem Ecology Systematics 27: 197-235.

Paine, R.T., Tegner, M.J. \& Johnson, E.A. (1998) Compounded perturbations yield ecological surprises. Ecosystems 1: 535-545.

Pearse, J.S. \& Hines, A.H. (1979) Expansion of a central California kelp forest following the mass mortality of sea urchins. Marine Biology 51: 83-91.

Peters, A.F. \& Breeman, A.M. (1993) Temperature tolerances and latitudinal range of brown algae from temperate Pacific South America. Marine Biology 115: 143-150.

Polis, G.A. \& Hurd, S.D. (1996) Linking marine and terrestrial food webs: allochthonous inputs from the ocean supports high secondary productivity on small islands and coastal land communities. American Naturalist 147: 396-523.

Pringle, J.D., Sharp, G.J. \& Caddy, J.F., eds. (1980) Proceedings of the Workshop on the Relationship Between Sea Urchin Grazing and Commercial Plant/Animal Harvesting. Canadian Technical Report of Fisheries and Aquatic Sciences, 954: 273 pp.

Raffaelli, D. \& Hawkins, S. (1996) Intertidal Ecology. London, UK: Chapman \& Hall.

Reed, D.C. \& Foster, M.S. (1984) The effects of canopy shading on algal recruitment and growth in a giant kelp forest. Ecology 65 : 937-948.

Rich, W.H. (1929) Fishing grounds of the Gulf of Maine. Bureau of Fisheries Documentary 1959: 51-117.

Rick, T.C., Erlandson, J.M. \& Vellanoweth, R. (2001) Paleocoastal marine fishing on the Pacific coast of the Americas: perspectives from Daisy Cave, California. American Antiquity 66: 593-613.

Rosier, J. (1605) True Relations of Captain George Weymouth's Voyage. London, UK: George Bishop.

Sala, E., Boudouresque, C.F. \& Harmelin-Viven, M. (1998) Fishing, trophic cascades, and the structure of algal assemblages: evaluation of an old but untested paradigm. Oikos 82: 425-439.

Salls, R.A. (1991) Early Holocene maritime adaptation at Eel Point, San Clemente Island. In: Hunter-Gathers Of Early Holocene Coastal California, ed. J.M. Erlandson \& R.H. Colton, pp. 63-80. Perspectives in California Archaeology, Volume I. Los Angeles, USA: Institute of Archaeology, University of California.

Salls, R.A. (1995) Ten-thousand years of fishing; the evidence for alternate stable states in nearshore ecosystems as the result of overexploitation of the California sheephead (Semiscossyphus 
pulcher) by prehistoric fishermen on San Clemente Island, California. In: Memorias IX Simposium Internacional Biologia Marina (June 1992), pp. 205-214. Universidad Autonoma Baja California Sur, La Paz, Baja California Sur, Mexico.

Santelices, B. (1990) Patterns of organizations of intertidal and shallow subtidal vegetation in wave exposed habitats of central Chile. Hydrobiologia 192: 35-57.

Santelices, B. \& Ojeda, F.P. (1984a) Effects of canopy removal on the understory algal community structure of coastal forests of Macrocystis pyrifera from southern South America. Marine Ecology Progress Series 14: 165-173.

Santelices, B. \& Ojeda, F.P. (1984b) Population dynamics of coastal forests of Macrocystis pyrifera in Puerto Toro, Isla Navarino, Southern Chile. Marine Ecology Progress Series 14: 175-183.

Scheffer, M., Carpenter, S., Foley, J.A., Folke, C. \& Walter, B. (2001) Catastrophic shifts in ecosystems. Nature 413: 591-596.

Scheibling, R.E. (1984) Echinoids, epizootics, and ecological stability in the rocky subtidal off Nova Scotia, Canada. Helgolander Wissenshaftlichen Meeresuntersuchungen 37: 233-242.

Scheibling, R.E. (1986) Increased macroalgal abundance following mass mortalities of sea urchins (Strongylocentrotus droebachiensis) along the Atlantic coast of Nova Scotia. Oecologia 68: 186-198.

Scheibling, R.E. \& Stephenson, R.L. (1984) Mass mortality of Strongylocentrotus droebachiensis (Echindermata: Echinoidea) off Nova Scotia, Canada. Marine Biology 78: 153-164.

Scheibling, R.E., Hennigar, A.W. \& Balch, T. (1999) Destructive grazing, epiphytism, and disease: the dynamics of sea urchin-kelp interactions in Nova Scotia. Canadian Fournal of Fisheries and Aquatic Sciences 56: 1-15.

Schiel, D.R. (1990) Macroalgal assemblages in New Zealand: structure, interactions and demography. Hydrobiologia 192: 59-76.

Schiel, D.R. \& Foster, M.S. (1986) The structure of subtidal algal stands in temperate waters. Oceanographic Marine Biology Annual Reviem 24: 265-307.

Schiel, D.R., Andrew, N.L. \& Foster, M.S (1995) The structure of subtidal algal and invertebrate assemblages at the Chatham Islands, New Zealand. Marine Biology 123: 355-367.

Schmitt, R.J. (1987) Indirect interactions between prey: apparent competition, predation, predator aggregation, and habitat segregation. Ecology 68: 1887-1897.

Seymour, R.J., Tegner, M.J., Dayton, P.K. \& Parnell, P.E. (1989) Storm wave induced mortality of giant kelp, Macrocystis pyrifera, in Southern California. Estuarine, Coastal and Shelf Science 28: 277-292.

Shepherd, S.A. \& Womersley, H.B.S. (1970) The sublittoral ecology of West Island, South Australia. I. Environmental features and the algal ecology. Transactions of the Royal Society of South Australia 94: 105-138.

Simenstad, C.A., Estes, J.A. \& Kenyon, K.W. (1978) Aleuts, sea otters, and alternate stable-state communities. Science 200: $403-411$.

Sivertsen, K. (1997). Geographic and environmental factors affecting the distribution of kelp beds and barren grounds and changes in biota associated with kelp reduction at sites along the Norwegian coast. Canadian Journal of Fisheries and Aquatic Sciences 54(12): 2872-2887.

Soares, A.G., Schlacher, T.A. \& McLachlan, A. (1997) Carbon and nitrogen exchange between sandy beach clams (Donax serra) and kelp beds in the Benguela coastal upwelling region. Marine Biology 127: 657-664.

Stachowicz, J.J. Whitlatch, R.B. \& Osman, R.W. (1999) Species diversity and invasion resistance in a marine ecosystem. Science 286: $1577-1579$.

Steele, J.H. (1998) Regime shifts in marine ecosystems. Ecological Applications 8: S33-S36.

Steneck, R.S. (1997) Fisheries-induced biological changes to the structure and function of the Gulf of Maine ecosystem. In: Proceedings of the Gulf of Maine Ecosystem Dynamics Scientific Symposium and Workshop, RARGOM Report 91-1, pp. 151-165. Hanover, NH, USA: Regional Association for Research in the Gulf of Maine.

Steneck, R.S. (1998) Human influences on coastal ecosystems: does overfishing create trophic cascades? Trends in Ecology and Evolution 13: 429-430.

Steneck, R.S. \& Carlton, J.T. (2001) Human alterations of marine communities: students beware! In: Marine Community Ecology, ed. M. Bertness, S. Gaines \& M. Hay, pp. 445-468. Sunderland, MA, USA: Sinauer Press.

Steneck, R.S. \& Dethier, M.N. (1994) A functional group approach to the structure of algal-dominated communities. Oikos 69 : 476-498.

Steneck, R.S. \& Watling, L.E. (1982) Feeding capabilities and limitations of herbivorous molluscs: a functional group approach. Marine Biology 68: 299-319.

Suzuki, T., Kuma, K., Kudo, I. \& Matsunaga, K. (1995) Iron requirement of the brown macroalgae Laminaria japonica, Undaria pinnatifida and the crustose coralline algae, and their competition in the northern Japan Sea. Phycologia 34: 201-205.

Tegner, M.J. \& Dayton, P.K. (1987) El Niño effects on southern California kelp forest communities. Advances in Ecological Research 17: 243-279.

Tegner, M.J. \& Dayton, P.K. (1991) Sea urchins, El Niños, and the long-term stability of Southern California kelp forest communities. Marine Ecology Progress Series 77: 49-63.

Tegner, M.J. \& Dayton, P.K. (2000) Ecosystem effects of fishing in kelp forest communities. ICES Fournal of Marine Science 57: 576-589.

Tegner, M.J. \& Levin, L.A. (1983) Spiny lobsters and sea urchins: analysis of a predator-prey interaction. Fournal of Experimental Marine Biology and Ecology 73: 125-150.

Tegner, M.J., Basch, L.V. \& Dayton, P.K. (1996b) Near extinction of an exploited marine invertebrate. Trends in Ecology and Evolution 11: 278-279.

Tegner, M.J., Dayton, P.K., Edwards, P.B. \& Riser, K.L. (1996a) Is there evidence for long-term climatic change in southern California kelp forests? California Cooperative Oceanic Fisheries Investigations Reports 37: 111-126.

Tegner, M.J., Dayton, P.K., Edwards, P.B. \& Riser, K.L. (1997) Large-scale, low-frequency oceanographic effects on kelp forest succession: a tale of two cohorts. Marine Ecology Progress Series 146: $117-134$.

Tegner, M.J., Dayton, P.K., Edwards, P.B., Riser, K.L., Chadwick, D.B., Dean, T.A. \& Deysher, L. (1995) Effects of large sewage spill on a kelp forest community: catastrophe or disturbance? Marine Environmental Research 40: 181-224.

Vadas, R.L. \& Steneck, R.S. (1988) Zonation of deep water benthic algae in the Gulf of Maine. Fournal of Phycology 24: 338-346.

Vadas, R.L. \& Steneck, R.S. (1995) Overfishing and inferences in kelp-sea urchin interactions. In: Ecology of Fjords and Coastal Waters, ed. H.R. Skjoldal, C. Hopkins, K.E. Erikstad \& H.P. Leinaas, pp. 509-524. Amsterdam, the Netherlands: Elsevier Science B.V. 
Vasquez, J.A. (1993) Abundance, distributional patterns and diets of main herbivorous and carnivorous species associated with Lessonia trabeculata kelp beds in northern Chile. Serie Ocasional Universidad Catolica del Norte 2: 213-229.

Vasquez, J.A. \& Buschmann, A.H. (1997) Herbivore-kelp interactions in Chilean subtidal communities: a review. Revista Chilena de Historia Natural 70: 41-52.

Vasquez, J.A., Castilla, J.C. \& Santelices, B. (1984) Distributional patterns and diets of four species of sea urchins in giant kelp forest (Macrocystis pyrifera) of Puerto Toro, Navarino Island, Chile. Marine Ecology Progress Series 19: 55-63.

Vavrinec, J. (2003) Resilience of green sea urchin (Strongylocentrotus droebachiensis) populations following fishing mortality: marine protected areas, larval ecology and post-settlement survival. Ph.D. dissertation, School of Marine Sciences, University of Maine, USA.

Velimirov, B., Field, F.G., Griffiths, C.L. \& Zoutendyk, P. (1977) The ecology of kelp bed communities in the Benguela upwelling system. Helgolander Wissenshaftlichen Meeresuntersuchungen 30: 495-518.

Vermeij, G.J. (2001) Community assembly in the sea: geologic history of the living shore biota. In: Marine Community Ecology, ed. M. Bertness, S. Gaines \& M. Hay, pp. 39-60. Sunderland, MA, USA: Sinauer Press.

Vetter, E.W. (1995) Detritus-based patches of high secondary production in the nearshore benthos. Marine Ecology Progress Series 120: 251-262.

Warton, W.G. \& Mann, K.H. (1981) Relationship between destruc- tive grazing by the sea urchin, Stronylocentrotus droebachiensis, and the abundance of American lobster, Homarus americanus, on the Atlantic coast of Nova Scotia. Canadian Fournal of Fisheries and Aquatic Sciences 38: 1339-1349.

Witman, J.D. (1987) Subtidal coexistence: storms, grazing, mutualism, and the zonation of kelps and mussels. Ecological Monographs 57: 167-187.

Witman, J.D. (1988) Stability of Atlantic kelp forests. Trends in Ecology and Evolution 3: 285-286.

Witman, J. \& Dayton, P. (2001) Rocky subtidal communities. In: Marine Community Ecology, ed. M. Bertness, S. Gaines \& M. Hay, pp. 339-366. Sunderland, MA, USA: Sinauer Press.

Yendo, K. (1902) Kaiso Isoyake Chosa Hokoku. Suisan Chosa Hokoku 12(1): 1-33. (In Japanese.)

Yendo, K. (1903) Investigations on 'Isoyake' (decrease of seaweed). Fournal of the Imperial Fisheries Bureau 12: 1-33. (In Japanese.)

Yendo, K. (1914) On the cultivation of seaweeds, with special accounts of their ecology. Economic Proceedings of the Royal Dublin Society 2(7): 105-122.

Zimmerman, R.C. \& Kremer, J.N. (1984) Episodic nutrient supply to a kelp forest ecosystem in Southern California. Fournal of Marine Research 42: 591-604.

Zimmerman, R.C. \& Kremer, J.N. (1986) In situ growth and chemical composition of the giant kelp, Macrocystis pyrifera: response to temporal changes in ambient nutrient availability. Marine Ecology Progress Series 27: 227-285.

Zobell, C.E. (1971) Drift seaweeds on San Diego county beaches. Nova Hedwigia 32: 269-314. 